\title{
Efficient Hybrid Density Functional Calculations for Large Periodic Systems Using Numerical Atomic Orbitals
}

\author{
Peize Lin, ${ }^{\dagger}$ Xinguo Ren, ${ }^{*, \dagger}$ and Lixin $\mathrm{He}^{*, \dagger}$ \\ $\dagger C A S$ Key Laboratory of Quantum Information, University of Science and Technology of \\ China, Hefei, Anhui 230026, China \\ $\ddagger$ Institute of Physics, Chinese Academy of Sciences, Beijing 100190, China \\ E-mail: renxg@iphy.ac.cn; helx@ustc.edu.cn
}

\begin{abstract}
We present an efficient, linear-scaling implementation for building the (screened) Hartree-Fock exchange (HFX) matrix for periodic systems within the framework of numerical atomic orbital (NAO) basis functions. Our implementation is based on the localized resolution of the identity approximation by which two-electron Coulomb repulsion integrals can be obtained by only computing two-center quantities - a feature that is highly beneficial to NAOs. By exploiting the locality of basis functions and efficient prescreening of the intermediate three- and two-index tensors, one can achieve a linear scaling of the computational cost for building the HFX matrix with respect to the system size. Our implementation is massively parallel, thanks to a MPI/OpenMP hybrid parallelization strategy for distributing the computational load and memory storage. All these factors add together to enable highly efficient hybrid functional calculations for large-scale periodic systems. In this work we describe the key algorithms and implementation details for the HFX build as implemented in the ABACUS code
\end{abstract}


package. The performance and scalability of our implementation with respect to the system size and the number of CPU cores are demonstrated for selected benchmark systems up to 4096 atoms.

\section{Introduction}

Hybrid density functionals (HDFs) ${ }^{1}$ belong to the fourth rung of the Jacob's ladder ${ }^{2}$ in KohnSham (KS) density functional theory (DFT). ${ }^{3,4}$ On the one hand, compared to lower-rung local- and semi-local approximations, HDFs, as implemented in the framework of generalized KS (gKS) theory, ${ }^{5}$ typically deliver better accuracy for ground-state properties and, additionally, provide physically more sound single-particle energy spectra. On the other hand, compared to the correlated methods (e.g., fifth-rung functionals, the quantum chemistry methods, and Green-function based many-body approaches), HDFs also have clear advantages. This is not only because HDFs are significantly cheaper than the correlated methods, but also because they are able to offer a range of useful properties in a single calculation, including e.g., electron density, ground-state energies, atomic structures, and single-particle orbital energies, and so on. Not all of these properties are easily accessible from the more expensive many-body approaches. As such, HDFs have been widely used in quantum chemistry, ${ }^{1,6-10}$ and are becoming increasingly popular in computational materials science. ${ }^{11}$

An essential feature of HDFs is that a portion of Hartree-Fock-type exchange (HFX) is incorporated in the construction of the exchange-correlation $(\mathrm{XC})$ functional. Furthermore, in recently developed HDFs, the range-separation framework ${ }^{12}$ is often invoked, in which the HFX is decomposed into a short-range part and a long-range part, and different portions of the two parts are utilized in the functional constructions. ${ }^{10,11,13-16}$ Another interesting development is that the initially empirical parameters in HDFs are made system-dependent and determined on the fly, ${ }^{16-22}$ or satisfying certain physical constrains. ${ }^{23,24}$ In particular, the concept of double screening mechanism involving both the dielectric screening and metallic

screening channel is instructive for developing more refined next-generation DHFs. ${ }^{17,18,20,25}$ 
All these efforts have increased the flexibility and application territories of HDFs. For a recent review, see Ref. ${ }^{26}$

Computationally, the build of the full-range or range-separated HFX matrix, constitutes the major bottleneck for HDF calculations. The canonical scaling of the computational cost of this step is $\mathcal{O}\left(N^{4}\right)$ with $N$ being the system size. Historically, efficient algorithms for computing the HFX matrix were first developed in quantum chemistry using atomcentered Gaussian-type orbitals (GTOs). Back then, $\mathcal{O}\left(N^{2}\right)$ scaling ${ }^{27,28}$ and even linearscaling algorithms ${ }^{29-33}$ for building the HFX matrix have been designed, by exploiting the locality of GTO basis functions, and (for insulating systems) the sparsity of the density matrix. To extend the application of HDFs to condensed matter systems, it is customary to impose the periodic boundary condition $(\mathrm{PBC})$ in the implementation. In practice, this means one needs to treat a rather large supercell, and handle additional complexities such as the translation symmetry with respect to the lattice vectors and the singularity of the Coulomb operator. Since the early periodic Hartree-Fock (HF) implementation ${ }^{34,35}$ in the CRYstal code, ${ }^{36}$ other GTO-based HF and HDF implementations have been reported, ${ }^{37-39}$ with linear-scaling cost for building the HFX matrix. As the interest in HDFs grows in the solid-state community, more HFD implementations have been reported, within the projector augmented wave (PAW), ${ }^{40}$ pseudopotential plane-wave (PW), and linearized augmented plane wave (LAPW $)^{41}$ frameworks, where the PBC is automatically satisfied. However, due to the extended nature of the PW and LAPW basis functions, the computational cost of the HFX in these implementations follows a canonical $\mathcal{O}\left(N^{4}\right)$ scaling as the system size. By a transformation from the Bloch orbitals to a localized Wannier function representation, numerical techniques can again be developed to achieve a linear-scaling numerical cost of the $\mathrm{HFX}^{42}$ matrix construction. Other techniques to speed up the HDF calculations have also been developed in the PW basis context, including, e.g., the recently proposed adaptively compressed exchange operator technique. ${ }^{43}$

In recent years, owing to their strict spatial locality and more realistic radial behavior, the 
numerical atomic orbitals (NAOs) are gaining increasing popularity as the basis set choice in first-principles electronic structure calculations.

For ground-state calculations based on conventional local and semilocal approximations, ${ }^{4,44-46}$ the reliability and efficiency of the NAO basis set have been well established, as been demonstrated by the flourishing of NAO-based first-principles computer code packages. $^{47-52}$

In case of HDFs, where the computation of two-electron Coulomb repulsion integrals (ERIs) is a necessity, NAO-based implementations have only appeared recently. Existing implementations circumvent a straightforward evaluation of ERIs in terms of NAOs in one way or another, by expanding the NAOs in terms of GTOs, ${ }^{53,54}$ or by employing the resolution-of-the-identity $(\mathrm{RI})$ technique $^{55-58}$ (as well as its refined variant - the interpolative separable density fitting scheme $\left.{ }^{59,60}\right)$. Within the $\mathrm{RI}^{61-63}$ - also known as variational density fitting ${ }^{64-66}$ - approximation, the four-index ERIs are decomposed into three- and two-index ones, whereby the storage requirement and the computational cost are significantly reduced. For molecular systems, the accuracy and efficiency of the conventional RI approximation based on the Coulomb metric have been well established for the HFX and explicit correlation calculations using both GTOs ${ }^{63,67-69}$ and NAOs. ${ }^{55}$

Despite its nice properties such as the preservation of positive definiteness of the ERI matrix and the vanishing of the expansion errors up to linear order, ${ }^{65,70}$ the conventional Coulomb-metric RI scheme is not particularly suitable for large molecules and extended systems. For these systems, the conventional RI scheme, which requires the computation of a very large number of three-center Coulomb integrals and an inversion of a large Coulomb matrix, can become prohibitively expensive. To deal with this problem, localized variants of the RI approach have been developed, ${ }^{56,70-76}$ where the products of two atomic orbitals (AOs) are expanded in terms of a limited set of auxiliary basis functions (ABFs) centering in the neighborhood of the two AO centers. Restricting the ABFs expanding the AO pair products to only the two center where the two AOs are located, one obtains what we called 
the localized RI (LRI) scheme, ${ }^{56-58}$ or the so-called pair-atomic RI (PARI) approximation ${ }^{70,77}$ in the quantum chemistry literature. The LRI scheme is essential for enabling efficient NAObased periodic HDF implementations. ${ }^{57,58}$

In a recent paper, ${ }^{58}$ we benchmarked the accuracy of LRI for HDF calculations within a pseudopotential-based NAO framework. Using the Heyd-Scuseria-Ernzerhof (HSE) screened $\mathrm{HDF}^{11}$ as implemented in the ABACUS (Atomic-orbital Based Ab-initio Computation at UStc) code, ${ }^{52,78,79}$ we showed that, when paired with suitably constructed auxiliary basis set, the errors in the computed band gap values incurred by LRI is below $10 \mathrm{meV}$ for prototypical semiconductors and insulators. Such an error is significantly smaller than the errors stemming from other sources, such as the incompleteness of one-electron basis set and the quality pseudopotentials. These tests indicate that, within the pseudopotential-based NAO framework, the LRI approximation is able to provide adequate accuracy for practical purposes.

In this paper, we describe the details of the HDF implementation within the ABACUS code package. ${ }^{52,78,79}$ ABACUS is a recently developed first-principles software that employs NAOs as basis functions and the norm-conserving pseudopotentials ${ }^{80}$ to describe the ion cores. An economic, linear-scaling implementation for building the HFX for large periodic systems has been carried out, utilizing several numerical algorithms including the LRI scheme to compute the ERIs, an efficient prescreening of the intermediate quantities to form the HFX matrix, an elegant "back-folding" procedure to account for the translational symmetry, and a "memorization" technique for efficiently handling the two-center Coulomb integrals. Furthermore, depending on the actual size of the system to be simulated and the available resources, we use either Machine-scheduling or K-means algorithm to distribute the computational load, resulting in a parallel program showing excellent efficiency up to thousands of CPU cores.

The outline of this paper is as follows: Section II briefly reviews the exact-exchange theory and the resolution of the identity scheme. Section III describes all algorithms and techniques 
we have proposed to decrease the computing time and memory consumes. Section IV shows the benchmark results about the accuracy and performance of the implementations. Section $\mathrm{V}$ concludes this paper.

\section{Methods}

In the present work, the HFX matrix is evaluated within the framework of linear combination of atomic orbitals (LCAO). Here, we explicitly consider periodic systems, and an AO $\phi_{i}(\mathbf{r})$ centering at an atom $\tilde{I}$ within a unit cell $\mathbf{R}_{I}$ is denoted as

$$
\phi_{\tilde{I}\left(\mathbf{R}_{I}\right) i}(\mathbf{r}) \stackrel{\text { def }}{=} \phi_{i}\left(\mathbf{r}-\boldsymbol{\tau}_{\tilde{I}}-\mathbf{R}_{I}\right)
$$

where $\boldsymbol{\tau}_{\tilde{I}}$ is the position of the atom $\tilde{I}$ within the unit cell. To simplify the notation, we omit the lattice vector index $\mathbf{R}_{I}$ in the following, and denote that $\phi_{\tilde{I}\left(\mathbf{R}_{I}\right) i}(\mathbf{r})=\phi_{I i}(\mathbf{r})$, but keep in mind that different atoms might be located in different unit cells. In the present paper, we choose to adopt a notational system that is slightly different from the one used in Ref. 58 , by which the indices of the atoms are clearly indicated. As will become clear later, by specifying the individual atoms from which the basis functions originate, our algorithm of evaluating the HFX matrix can be better explained.

Within the LCAO formulation, the HFX matrix for a periodic system is formally given by

$$
\Sigma_{I i, J j}^{\mathrm{X}}=\sum_{K, L} \sum_{k \in K, l \in L}\left(\phi_{I i} \phi_{K k} \mid \phi_{J j} \phi_{L l}\right) D_{K k, L l}
$$

where we use the abbreviation

$$
(f \mid g) \stackrel{\text { def }}{=} \iint f(\mathbf{r}) v\left(\mathbf{r}-\mathbf{r}^{\prime}\right) g\left(\mathbf{r}^{\prime}\right) d \mathbf{r} d \mathbf{r}^{\prime}
$$

to denote the interaction integral between two functions $f(\mathbf{r})$ and $g(\mathbf{r})$ via a potential $v\left(\mathbf{r}-\mathbf{r}^{\prime}\right)$ 
and $D_{K k, L l}$ is the density matrix in real space,

$$
D_{K k, L l}=\frac{1}{N_{\mathbf{k}}} \sum_{n, \mathbf{k} \in 1 \mathrm{BZ}} f_{n \mathbf{k}} c_{k, n}(\mathbf{k}) c_{l, n}^{*}(\mathbf{k}) e^{-i \mathbf{k} \cdot\left(\mathbf{R}_{K}-\mathbf{R}_{L}\right)}
$$

In eq $(4), c_{k, n}(\mathbf{k})$ are KS eigenvectors, $f_{n \mathbf{k}}$ are the occupation numbers, and $N_{\mathbf{k}}$ is the number of $\mathbf{k}$ points in the 1st Brillouin zone (1BZ). Furthermore, the lattice vectors $\mathbf{R}_{K}$ and $\mathbf{R}_{L}$ label the unit cells where the atom $K$ and $L$ are located, respectively. Depending on the choice of the potential $v\left(\mathbf{r}-\mathbf{r}^{\prime}\right)$ in eq (3), one can either obtain the full-range HFX (when $\left.v\left(\mathbf{r}-\mathbf{r}^{\prime}\right)=1 /\left|\mathbf{r}-\mathbf{r}^{\prime}\right|\right)$ or the short-range $\operatorname{HFX}\left(\right.$ e.g., $v\left(\mathbf{r}-\mathbf{r}^{\prime}\right)=\operatorname{erfc}\left(\mu\left|\mathbf{r}-\mathbf{r}^{\prime}\right|\right) /\left|\mathbf{r}-\mathbf{r}^{\prime}\right|$, with $\operatorname{erfc}(x)$ being the complementary error function, and $\mu$ a range-separation parameter). Since our algorithm presented below does not depend on the specific form of $v\left(\mathbf{r}-\mathbf{r}^{\prime}\right)$, we don't distinguish full-range or short-range HFX, unless this turns out to be necessary.

In this work, we use the LRI approximation, ${ }^{56-58}$ also known as PARI, ${ }^{70,77}$ to evaluate the ERIs. Within LRI, the product of two NAOs centering on two atoms is approximately expanded as

$$
\begin{aligned}
\phi_{I i}(\mathbf{r}) \phi_{K k}(\mathbf{r}) & \approx \sum_{A=\{I, K\}} \sum_{\alpha \in A} C_{I i, K k}^{A \alpha} P_{A \alpha}(\mathbf{r}) \\
& =\sum_{\alpha \in I} C_{I i, K k}^{I \alpha} P_{I \alpha}(\mathbf{r})+\sum_{\alpha \in K} C_{K k, I i}^{K \alpha} P_{K \alpha}(\mathbf{r})
\end{aligned}
$$

where, similar to the AO case (eq (1)),

$$
P_{A \alpha}(\mathbf{r}) \stackrel{\text { def }}{=} P_{\tilde{A}\left(\mathbf{R}_{A}\right) \alpha}(\mathbf{r})=P_{\alpha}\left(\mathbf{r}-\boldsymbol{\tau}_{\tilde{A}}-\mathbf{R}_{A}\right)
$$

and $C_{I i, K k}^{A \alpha}$ with $A=\{I, K\}$ are the two-center, three-index expansion coefficients. The lower (Roman) and upper (Greek) indices of the $C$ coefficients denote the AOs and ABFs, respectively. The essence of eq (5) is that the ABFs are restricted either on the atom $I$ or the atom $K$, on which the two AOs are centering. It should be noted that, the expansion 
coefficients $C$, although carrying three orbital indices, can be obtained in terms of two-center integrals, ${ }^{56,58}$ which may be evaluated rather efficiently using the algorithm developed by Talman $^{81-83}$ for NAOs.

Using eq (5), the computational expression of the HFX in eq (2) naturally splits into four pieces,

$$
\begin{aligned}
\Sigma_{I i, J j}^{\mathrm{x}} \approx \sum_{K, L} \sum_{k \in K, l \in L} & {\left[\sum_{\alpha \in I, \beta \in J} C_{I i, K k}^{I \alpha}\left(P_{I \alpha} \mid P_{J \beta}\right) C_{J j, L l}^{J \beta}+\sum_{\alpha \in I, \beta \in L} C_{I i, K k}^{I \alpha}\left(P_{I \alpha} \mid P_{L \beta}\right) C_{J j, L l}^{L \beta}\right.} \\
& \left.\sum_{\alpha \in K, \beta \in J} C_{I i, K k}^{K \alpha}\left(P_{K \alpha} \mid P_{J \beta}\right) C_{J j, L l}^{J \beta}+\sum_{\alpha \in K, \beta \in L} C_{I i, K k}^{K \alpha}\left(P_{K \alpha} \mid P_{L \beta}\right) C_{J j, L l}^{L \beta}\right] D_{K k, L l}
\end{aligned}
$$

where $\left(P_{A \alpha} \mid P_{B \beta}\right)$ with $A=\{I, K\}$ and $B=\{J, L\}$ are the (screened) Coulomb integrals between two ABFs, defined by eq (3). For notational convenience, below we also denote the (screened) Coulomb integrals as $V_{A \alpha, B \beta} \stackrel{\text { def }}{=}\left(P_{A \alpha} \mid P_{B \beta}\right)$.

In order to present our algorithm in a clear way, we re-express eq (7) as

$$
\Sigma_{I i, J j}^{\mathrm{X}} \approx \sum_{K, L}\left[\mathcal{H}_{\underline{I}, K \mid \underline{J j}, L}^{\mathrm{X}}+\mathcal{H}_{\underline{I}, K \mid J j, \underline{L}}^{\mathrm{X}}+\mathcal{H}_{I i, \underline{K} \mid \underline{j} j, L}^{\mathrm{X}}+\mathcal{H}_{I i, \underline{K} \mid J j, \underline{L}}^{\mathrm{X}}\right]
$$

where each of four terms in eq (8) stems from a corresponding one in eq (7), e.g.,

$$
\begin{aligned}
\mathcal{H}_{\underline{I} i, K \mid \underline{J} j, L}^{\mathrm{X}} & \stackrel{\text { def }}{=} \sum_{k \in K, l \in L} \sum_{\alpha \in I, \beta \in J} C_{I i, K k}^{I \alpha} V_{I \alpha, J \beta} C_{J j, L l}^{J \beta} D_{K k, L l} \\
& =\sum_{k \in K, l \in L}\left[\phi_{\underline{I}} \phi_{K k} \mid \phi_{\underline{J j}} \phi_{L l}\right] D_{K k, L l}
\end{aligned}
$$

with the underlined atomic indices highlighting those two atoms on which the ABFs are located. Furthermore, for the $\mathcal{H}^{\mathrm{x}}$ objects, the atoms $K$ and $L$ don't carry AO indices $k, l$ since they have been contracted out with the density matrix. In eq (9), we have introduced 
partial two-electron Coulomb integrals denoted by square brackets,

$$
\left[\phi_{\underline{A} a} \phi_{F f} \mid \phi_{\underline{B} b} \phi_{G g}\right] \stackrel{\text { def }}{=} \sum_{\alpha \in A, \beta \in B} C_{A a, F f}^{A \alpha} V_{A \alpha, B \beta} C_{B b, G g}^{B \beta}
$$

where $A, B, F, G$ are atomic indices and $a, b, f, g$ are AOs centering on these atoms. Again, the underscored indices for atoms $A$ and $B$ indicated these are the atoms where the ABFs are located. Note that exchanging the positions of $A$ and $F$, or $B$ and $G$ in the square bracket does not change the value of the integral, namely, $\left[\phi_{\underline{A} a} \phi_{F f} \mid \phi_{\underline{B} b} \phi_{G g}\right]=\left[\phi_{F f} \phi_{\underline{A} a} \mid \phi_{\underline{B} b} \phi_{G g}\right]=$ $\left[\phi_{\underline{A} a} \phi_{F f} \mid \phi_{G g} \phi_{\underline{B} b}\right]=\left[\phi_{F f} \phi_{\underline{A} a} \mid \phi_{G g} \phi_{\underline{B} b}\right]$. In this work, the capital letters $I, J$ are reserved for the atoms to which the $\mathrm{AO}$ indices of the HFX matrix belong, $K, L$ for the atoms to the AO indices of the density matrix belong, whereas $A, B$ are referred to the atoms on which the ABFs are sitting, and $F, G$ for the atoms without ABFs. According to the definition given by eq (10), one can easily see that the full ERIs are given by the sum of four partial ERIs,

$$
\left(\phi_{I i} \phi_{K k} \mid \phi_{J j} \phi_{L l}\right) \approx\left[\phi_{\underline{I} i} \phi_{K k} \mid \phi_{\underline{J} j} \phi_{L l}\right]+\left[\phi_{\underline{I} i} \phi_{K k} \mid \phi_{J j} \phi_{\underline{L} l}\right]+\left[\phi_{I i} \phi_{\underline{K} k} \mid \phi_{\underline{J} j} \phi_{L l}\right]+\left[\phi_{I i} \phi_{\underline{K} k} \mid \phi_{J j} \phi_{\underline{L l}}\right] .
$$

However, as detailed below, in our practical implementations, we never need to form the full ERIs according to eq (11). Rather, the $\mathcal{H}^{\mathrm{X}}$ objects, given by the contraction of the partial ERIs with the density matrix, are used to build the HFX matrix. This choice is made for the sake for designing highly efficient parallel algorithms.

Equations (8)-(10) serve as the mathematical foundation for one to design efficient practical algorithms to evaluate the HFX matrix. We remark that the error incurred by LRI, as given by eq (5), can be made sufficiently small, provided that suitable auxiliary basis sets can be constructed. The accuracy aspect of our HDF implementation based on LRI has been benchmarked in a previous paper. ${ }^{58}$ In the present paper, we focus on the implementation details, as well as the efficiency and the scaling behavior of the implementation. 


\section{Implementation Details}

As mentioned above, the key underlying equations behind our implementation are eqs (8)(10), on which our algorithm for building the HFX matrix is based. To achieve good efficiency and scalability, specific details need to be considered, such as how to design the loop structure, how to screen out the ERIs below a pre-given threshold, and how to distribute the computational load and memory storage over the MPI (Message Passing Interface ${ }^{84}$ ) tasks. These details will be discussed in the present section.

\subsection{Loop structure for evaluating the HFX matrix}

The loop structure for a straightforward evaluation of the HFX matrix is presented in Algorithm 1. In this algorithm, one first loops over the atomic indices of the HFX matrix, i.e., the atom pair $\langle I, J\rangle$. Here the translational symmetry of periodic systems can be taken into account by restricting the atom $I$ within the central unit cell (i.e., setting $\mathbf{R}_{I}=\mathbf{0}$ ), while allowing the atom $J$ to be placed in the entire supercell. For each atom pair $\langle I, J\rangle$, one goes through all the atoms $K$ in the neighborhood of the atom $I$ (denoted as $K \in \mathfrak{N}[I]$ ), and all the atoms $L$ in the neighborhood of $J$ (i.e., $L \in \mathfrak{N}[J]$ ). Within the loop over atoms $K$ and $L$, one can form all the partial ERIs belonging to these four atoms, namely, $\left[\phi_{\underline{I}} \phi_{K k} \mid \phi_{\underline{J} j} \phi_{L l}\right]$, $\left[\phi_{\underline{I} i} \phi_{K k} \mid \phi_{J j} \phi_{\underline{L} l}\right],\left[\phi_{I i} \phi_{\underline{K} k} \mid \phi_{\underline{J j}} \phi_{L l}\right],\left[\phi_{I i} \phi_{\underline{K} k} \mid \phi_{J j} \phi_{\underline{L l}}\right]$. However, one may choose not to form the full ERIs according to eq (11) here. This is because, in parallel computing, these partial ERIs are evaluated and stored on different processes, and forming the full ERIs requires a significant amount of communication. Instead, they are contracted locally with the density matrix, ending up with the much smaller $\mathcal{H}^{\mathrm{x}}$ objects, as illustrated in Algorithm 1. Adding up these $\mathcal{H}^{\mathrm{X}}$ objects for all $\langle K, L\rangle$ atom pairs, one obtains the full desired HFX matrix.

Such a straightforward calculation flow works, but is not an efficient one. In fact, in Algorithm 1, each independent partial ERI has been computed four times, which is a big waste. For example, as noted above, the partial ERI $\left[\phi_{\underline{I i}} \phi_{K k} \mid \phi_{\underline{J j}} \phi_{L l}\right]$ is equal to $\left[\phi_{K k} \phi_{\underline{I} i} \mid \phi_{\underline{J j}} \phi_{L l}\right]$ due 
to the presence of permutation symmetry, but both need to be computed in Algorithm 1. The same happens when one swaps the $\underline{J}$ and $L$ indices. This is because these partial ERIs cannot be stored in memory at the same time, and hence they are computed on the fly within the $\langle I, J\rangle$ loop. As a consequence, only partial ERIs with a fixed $\langle I, J\rangle$ pair are available at a time. Thus for $I \neq K,\left[\phi_{K k} \phi_{\underline{I}} \mid \phi_{\underline{J} j} \phi_{L l}\right]$ always needs to computed, since its equivalent $\left[\phi_{\underline{I} i} \phi_{K k} \mid \phi_{\underline{J j}} \phi_{L l}\right]$ is not available any, even if it has been computed before.

To overcome this redundancy issue, we switch to a revised loop structure as presented in Algorithm 2. A key feature of Algorithm 2 is that each independent partial ERI is computed only once. To this end, instead of first looping over the indices of the HFX matrix, i.e., the $\langle I, J\rangle$ pair, we loop over the $\langle A, B\rangle$ pair, on which the ABFs are located. (Again, the translation symmetry can be accounted by restricting $A$ in the central unit cell, while allowing $B$ to be located in the entire supercell.) Within the $\langle A, B\rangle$ loop, one computes and stores the partial ERIs $\left[\phi_{\underline{A} a} \phi_{F f} \mid \phi_{\underline{B} b} \phi_{G g}\right]$ for all $F \in \mathfrak{N}[A]$ and $G \in \mathfrak{N}[B]$. To maximize the usage of the partial ERIs that are present at a time, we contract the fourth-rank tensor $\left[\phi_{\underline{A} a} \phi_{F f} \mid \phi_{\underline{B} b} \phi_{G g}\right]$ for a given set of atoms $A, B, F, G$ with the density matrix $D_{F f, G g}, D_{F f, B b}$, $D_{A a, G g}$, and $D_{A a, B b}$, ending up with contributions to different sectors of the HFX matrix, as illustrated in Algorithm 2. In this algorithm, the permutation symmetry of the ERIs is naturally incorporated, and each independent partial ERI is computed only once. The trick here is that, with the new loop structure, we can contract the partial ERIs with different subblocks of the density matrix originating from different pairs of atoms. When the loop over $\langle A, B\rangle$ is complete, the full HFX matrix is obtained. This redesign of the loop structure speeds up the calculations by roughly a factor of 4 .

In addition to improved efficiency, Algorithm 2 is also advantageous for memory usage in case of parallel computing. To understand this point, we note that the atom pairs at the outermost loop are distributed over the MPI processes. Specifically, in Algorithm 1, the $\langle I, J\rangle$ pairs are distributed, and for a given $\langle I, J\rangle$ pair, the information for $\forall K \in \mathfrak{N}[I]$ and $\forall L \in \mathfrak{N}[J]$ is stored locally. These include subblocks of both the Coulomb matrix $V_{K \mu, L \nu}$ 

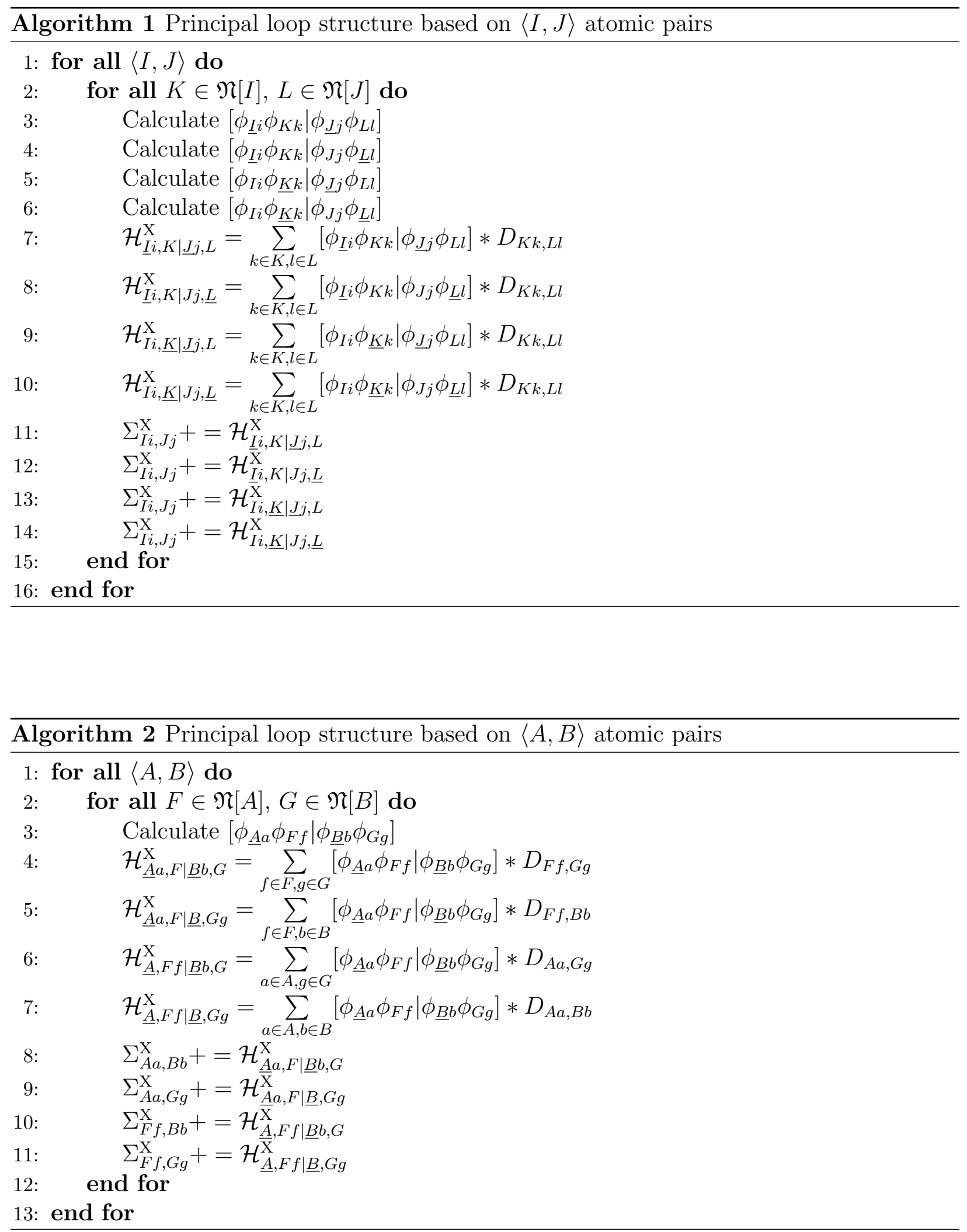
and the density matrix $D_{K k, L l}$ originating from $K$ and $L$ atoms. Since the same atom pairs $\langle K, L\rangle$ can be neighbors of different $\langle I, J\rangle$ pairs, there will be a duplication of $\langle K, L\rangle$ atom pairs, and hence a duplication of $V_{K \alpha, L \beta}$ and $D_{K k, L l}$ matrices over MPI processes, but there is no duplication of the $\Sigma_{I i, J j}^{\mathrm{X}}$ matrix. Following a similar line of reasoning, in Algorithm 2, $D_{F f, G g}$ and $\Sigma_{F f, G g}^{\mathrm{X}}$ matrices are duplicated, but there is no duplication of $V_{A \alpha, B \beta}$ matrix. Since the size of ABFs is several times larger than that of the AOs, the size of the $V$ is much larger than that of the $\Sigma^{\mathrm{X}}$ matrix. Thus Algorithm 2 consumes less memory than Algorithm 1 and this is the second advantage of Algorithm 2.

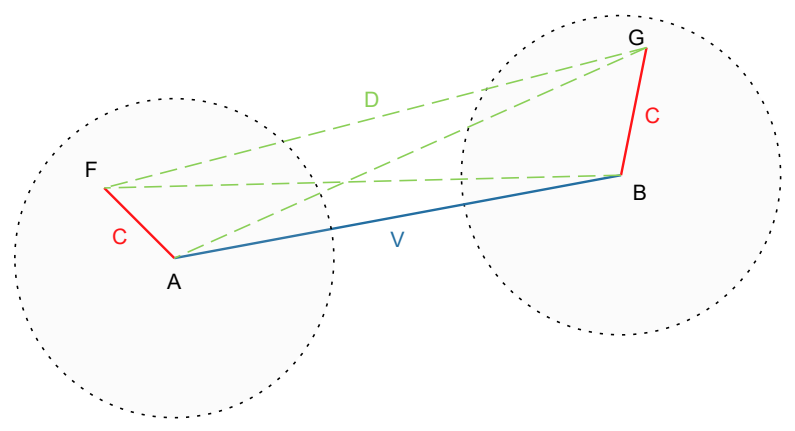

Figure 1: Pictorial illustration of Algorithm 2. The four dots denote four atoms $A, B, F$ and $G$, where $A$ and $B$ are the atoms where the ABFs are located, and hence they are connected by a $V$ (interaction) line. The atoms $F$ and $G$ sit in the neighborhood of $A$ and $B$, respectively, represented by two circles centered at $A$ and $B$. The atoms $A$ and $F$, as well as $B$ and $G$ are connected by the $C$ (expansion coefficient) lines. The green dash lines denote the density matrix between two atoms. For periodic systems the atom $A$ is restricted to the central unit cell, whereas the atom $B$ is located in the entire supercell.

A pictorial illustration of Algorithm 2 is presented in Fig. 1, where the atoms are denoted by dots and the expansion coefficients $C$, the Coulomb matrix $V$, and the density matrix $D$ are represented by lines. Finally we remark that the flowcharts presented in Algorithm 1 and 2 are mainly used to elucidate our choice of the major loop structure of our implementation. In practical implementation, it is in fact not necessary (and too expensive) to explicitly form the partial ERIs $\left[\phi_{\underline{A} a} \phi_{F f} \mid \phi_{\underline{B b}} \phi_{G g}\right]$. As shown in Appendix A.1, the most efficient way to proceed is to first contract the Coulomb matrix $V$ with the expansion coefficients $C$, and then multiply the resultant quantity with the density matrix $D$. At a final step, the HFX 
matrix by multiply $C$ with the product of $(V C)$ and $D$. Namely, $\Sigma^{\mathrm{X}} \sim C((V C) D)$. An illustration of this refined procedure adopted in our practical implementation is given in Algorithm 3.

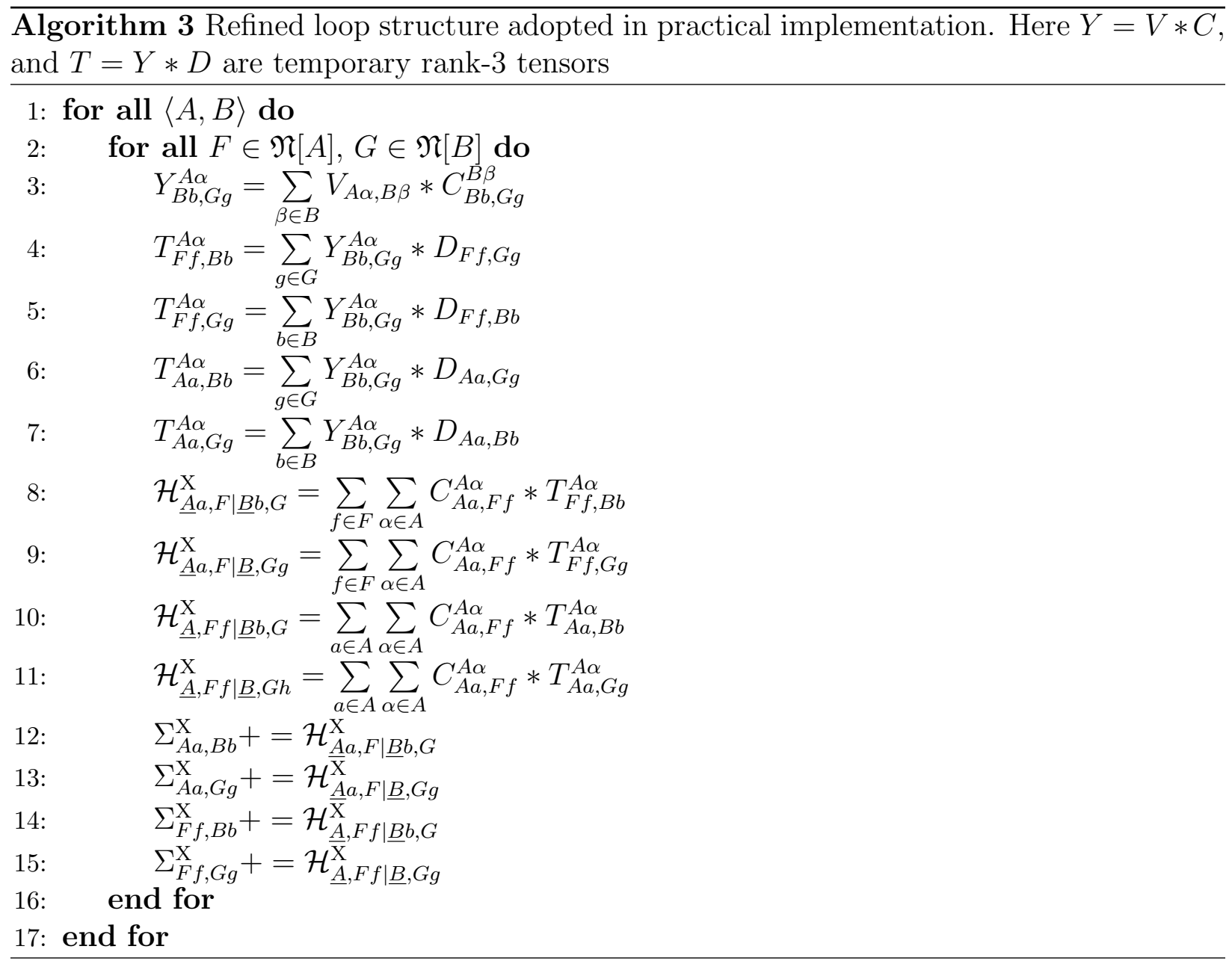

\subsection{Sparsity of matrices and pre-screening}

As discussed above, the most time-consuming step in self-consistent HDF calculations is to construct the HFX matrix. The building blocks of the HFX matrix is the $\mathcal{H}^{\mathrm{X}}$ objects, introduced in eqs (8) and (9), which are given schematically by $\mathcal{H}^{\mathrm{x}}=C V C D$, where $C, V$, and $D$ denote, respectively, the expansion coefficient matrix (eq (5)), the (screened) Coulomb matrix, and the density matrix. A straightforward evaluation of all $\mathcal{H}^{\mathrm{X}}$ objects scales as $N_{\mathrm{at}}^{4}$, where $N_{\text {at }}$ is the number of atoms in the system. However, due to the locality of the NAOs 
and, in case of insulators, the rapidly decaying behavior of the density matrix in real space, only the exchange interactions within a limited spatial range are needed. In fact, a large portion of elements of the matrix $\mathcal{H}^{\mathrm{x}}$, as well as those of the $C, V$ and $D$ matrices are extremely small or even strictly zero. Eliminating these small matrix elements has little effect on the obtained results, but can save a lot of computation time and memory. As will be shown below, by exploiting the sparsity of these matrices, one can achieve linear scaling of the computational cost for evaluating the $\Sigma^{\mathrm{X}}$ matrices, and hence a linear-scaling build of the HFX matrix.

\subsubsection{The matrix of the expansion coefficients $C$}

Let's first look at the $C$ matrix. Because the NAOs have a finite cut-off radius, their overlap and consequently the expansion coefficients $C_{A a, F f}^{A \alpha}$ become strictly zero if the distance between two atoms $A$ and $F$ is larger than the sum of the cut-off radii of AO basis functions $a$ and $f$, as illustrated in Fig. 1. When discussing Algorithm 1 and 2 we already introduced the notation of neighboring atoms. Here we shall define that the neighboring atoms $F$ of an atom $A$ are those for which the expansion coefficients $C_{A a, F f}^{A \alpha}$ are non-zero, i.e., $\mathfrak{N}[A] \stackrel{\text { def }}{=}\left\{F \mid C_{A a, F f}^{A \alpha} \neq 0, \forall a \in A, f \in F, \alpha \in A\right\}$. The matrix elements of $C$ originating from atom pairs that are not "neighbors" are strictly zero and are excluded from the outset

of the calculations. In addition, there are $C_{A a, F f}^{A \alpha}$ elements which are sufficiently small to be negligible, without affecting the obtained results. As detailed below, these can be filtered out to further save computation time and memory.

\subsubsection{The (screened) Coulomb matrix $V$}

The matrix elements of the (screened) Coulomb interaction between the ABFs are calculated as,

$$
\begin{aligned}
V_{A \alpha, B \beta} & =\left(P_{A \alpha} \mid P_{B \beta}\right) \\
& =\left\langle P_{A \alpha} \mid Q_{B \beta}\right\rangle
\end{aligned}
$$


where $Q_{B \beta}(\mathbf{r})=\int v\left(\mathbf{r}-\mathbf{r}^{\prime}\right) P_{B \beta}\left(\mathbf{r}^{\prime}\right) d \mathbf{r}^{\prime}$, and $\langle f \mid g\rangle$ denotes the overlap integral between two functions $f(\mathbf{r})$ and $g(\mathbf{r})$.

For the bare Coulomb potential $v\left(\mathbf{r}, \mathbf{r}^{\prime}\right)=1 /\left|\mathbf{r}-\mathbf{r}^{\prime}\right|, Q_{\beta}(\mathbf{r})$ is has a rather long range and the sparsity of the $V$ matrix is low. In fact, in this case certain elements in the Coulomb matrix become diverging at the $\Gamma$ point when Fourier transformed to $\mathbf{k}$ space. Well-established procedures exist to deal with this so-called $\Gamma$-point singularity ${ }^{57,85,86}$ and we don't discuss this issue in the present paper. For screened HDFs such as HSE, one employs the shortranged (screened) Coulomb interaction $v\left(\mathbf{r}, \mathbf{r}^{\prime}\right)=\operatorname{erfc}(\mu|\mathbf{r}-\mathbf{r}|) /|\mathbf{r}-\mathbf{r}|$, and hence $Q_{\beta}(\mathbf{r})$ is short ranged. In this case, one can introduce a finite cut-off radius $R_{Q}$ for $Q_{\beta}(\mathbf{r})$, beyond which the elements of the (screened) Coulomb matrix become sufficiently small and can be neglected. Of course the sparsity of the obtained $V$ matrix depends on the screening parameter $\mu$.

\subsubsection{The density matrix $D$}

The density matrix $D_{K k, L l}$ of an insulator has an exponential decay behavior as a function of the distance between the two atoms. ${ }^{87-89}$ Therefore, if two atoms are far apart, the value of $D_{K k, L l}$ may be small enough to be negligible. However, for metallic systems, the density matrix only has a polynomial delay, and its sparsity is much reduced. Similar to the case of $C$, the insignificant elements of $D$ are also filtered out, as detailed below.

\subsection{4 $\mathcal{H}^{\mathrm{X}}$ matrices}

The $\mathcal{H}^{\mathrm{x}}$ matrices are given by the product of $C, V$, and $D$, and hence their sparsity originates from these individual matrices. To exploit the sparsity of these matrices and to make the best use of $\mathrm{BLAS}^{90-92}$ matrix multiplications which are most suitable for dense matrices, an optimal strategy is to organize and store the elements of the these matrices as sparsely distributed dense subblocks. With this in mind, in our implementation the data structure of these four tensors are all stored as aggregates of small block tensors. For example, the global 
matrix $C_{A a, F f}^{A \alpha}$ can be represented as a supermatrix $C_{A, F}$, which itself is a sparse matrix, while its non-zero elements are dense rank-3 tensors $C_{a, f}^{\alpha}$. Similarly, the global $V$ and $D$ matrices can be seen as atom-pair based supermatrices $V_{A, B}$ and $D_{K, L}(K=\{A, F\}, L=\{B, G\}$, see Algorithm 2), with each element of them being a rank-2 tensor $V_{\alpha, \beta}$ and $D_{k, l}$, respectively. To exploit the sparsity of $C$ mentioned in 3.2.1, an upper limit

$$
\overline{C_{A, F}} \stackrel{\text { def }}{=} \max _{a \in A, f \in F, \alpha \in A}\left|C_{A a, F f}^{A \alpha}\right|
$$

for each block $C_{A, F}$ is introduced. In practical implementation, one can introduce a finite threshold parameter $\varepsilon_{C}$. If $\overline{C_{A, F}} \leq \varepsilon_{C}$, the entire block $C_{A, F}$ (i.e., the contribution from the $\langle A, F\rangle$ atom pair) is disregarded. Similarly, an upper bound $\overline{D_{K, L}}$ can be defined for each block $D_{K, L}$, and the density matrix blocks with $\overline{D_{K, L}} \leq \varepsilon_{D}$ are discarded. The filtering of negligible blocks of the $V$ matrix is controlled by the cut-off radius $R_{Q}$. The influence of $\varepsilon_{C}$, $\varepsilon_{D}$, and $R_{Q}$ on the accuracy of the obtained results and the computational efficiency will be discussed in Sec. 4.1.1.

Due to the strict locality of the supermatrix $C_{A, F}\left(C_{B, G}\right)$, the atom $F(G)$ is constrained to the neighbouring atoms of $A(B)$. Furthermore, as long as either the Coulomb matrix (in case of screened HDFs ) or density matrix (in case of insulators) is short-ranged, only atom pairs $\langle A, B\rangle$ within a certain range contribute to the $\mathcal{H}^{\mathrm{X}}$ matrices. In other words, for a given atom $A$, the numbers of neighbouring atoms $B, F$ and $G$ contributing to the final HFX matrix are independent of system size, which warrants a linear-scaling computational cost for evaluating the HFX matrix, at least for screened HDFs or for insulating systems.

According to the formal relationship $\mathcal{H}^{\mathrm{x}} \sim C V C D$, it is obvious that the sparsity of $\mathcal{H}^{\mathrm{X}}$ comes from two aspects. For one thing, the zeros of individual blocks $C_{A, F}, V_{A, B}$, and $D_{K, L}$ directly lead to the sparsity of the $\mathcal{H}^{\mathrm{X}}$ matrices. For example, for a given set of atoms $A, B, F, G$, the matrix block $\mathcal{H}_{\underline{A} a, F \mid \underline{B} b, G}^{\mathrm{X}}$ is non-zero only if the four multiplier matrix blocks are all non-zero. Another possibility is that, even if the elements of the component matrices 
exceed their own screening thresholds, certain elements of the resultant product matrix $\mathcal{H}^{\mathrm{X}}$ may still be negligibly small. These small matrix elements can be efficiently screened out by making use of the Cauchy-Schwarz inequalities. The detailed screening procedures will be discussed in Sec. 3.3.1 and Sec. 3.3.2.

\subsection{The screening algorithm}

As discussed above, even after pre-screening the individual $C, V$, and $D$ matrices, there is still a portion of the $\mathcal{H}^{\mathrm{x}}$ matrix elements being extremely small, and can be safely neglected without affecting the obtained results. Thus, it would be highly desirable if the insignificant elements of the $\mathcal{H}^{\mathrm{x}}$ matrices can be efficiently filtered out, without actually calculating them. This is achieved by estimating the upper bounds of these matrix elements based on the Cauchy-Schwarz inequality, as detailed below, and neglecting the elements below a pre-chosen threshold at the early stage of the calculations.

\subsubsection{Cauchy-Schwarz inequality matrix screening}

The upper bounds of the matrix elements of $\mathcal{H}^{\mathrm{x}}$ are estimated in terms of Cauchy-Schwarz inequality, according to which the product of two matrices $\mathcal{A}$ and $\mathcal{B}$ satisfies

$$
|\operatorname{tr}[\mathcal{A B}]| \leq \sqrt{\operatorname{tr}\left[\mathcal{A}^{+} \mathcal{A}\right]} \sqrt{\operatorname{tr}\left[\mathcal{B}^{+} \mathcal{B}\right]}=\|\mathcal{A}\|\|\mathcal{B}\|
$$

where $\|\mathcal{A}\|=\sqrt{\sum_{i j}\left|a_{i j}\right|^{2}}$ is the L2-norm of the matrix $\mathcal{A}$. The Cauchy-Schwarz inequality can be extended straightforwardly to the multiplication of three and more matrices, e.g.,

$$
\begin{aligned}
& |\operatorname{tr}[\mathcal{A B C}]| \\
\leq & \sqrt{\operatorname{tr}\left[\mathcal{A}^{+} \mathcal{A}\right]} \sqrt{\operatorname{tr}\left[(\mathcal{B C})^{+}(\mathcal{B C})\right]} \\
= & \|\mathcal{A}\| \sqrt{\operatorname{tr}\left[\left(\mathcal{B}^{+} \mathcal{B}\right)(\mathcal{C C}+)\right]} \\
\leq & \|\mathcal{A}\| \sqrt{\left\|\mathcal{B}^{+} \mathcal{B}\right\|} \sqrt{\left\|\mathcal{C C}^{+}\right\|}
\end{aligned}
$$


and

$$
\begin{aligned}
& |\operatorname{tr}[\mathcal{A B C D}]| \\
\leq & \sqrt{\operatorname{tr}\left[(\mathcal{A B})^{+}(\mathcal{A B})\right]} \sqrt{\operatorname{tr}\left[(\mathcal{C D})^{+}(\mathcal{C D})\right]} \\
= & \sqrt{\operatorname{tr}\left[\left(\mathcal{A}^{+} \mathcal{A}\right)\left(\mathcal{B B}^{+}\right)\right]} \sqrt{\operatorname{tr}\left[\left(\mathcal{C}^{+} \mathcal{C}\right)\left(\mathcal{D D}^{+}\right)\right]} \\
\leq & \sqrt{\left\|\mathcal{A}^{+} \mathcal{A}\right\|} \sqrt{\left\|\mathcal{B B}^{+}\right\|} \sqrt{\left\|\mathcal{C}^{+} \mathcal{C}\right\|} \sqrt{\left\|\mathcal{D D}^{+}\right\|}
\end{aligned}
$$

Now we can apply the Cauchy-Schwarz inequality to eq (9) for a fixed set of atoms $I, J, K, L$, and obtain (symbolically)

$$
\begin{aligned}
\mathcal{H}_{\underline{I}, K \mid \underline{J} j, L}^{\mathrm{X}} & =\sum_{k \in K, l \in L} \sum_{\alpha \in I, \beta \in J} & & C_{I i, K k}^{I \alpha} V_{I \alpha, J \beta} C_{J j, L l}^{J \beta} D_{K k, L l} \\
& =\operatorname{tr}\left[C_{i} V C_{j} D\right] & & \left(\leq \sqrt{\left\|C_{i} C_{i}^{+}\right\|} \sqrt{\left\|V V^{+}\right\|} \sqrt{\left\|C_{j} C_{j}^{+}\right\|} \sqrt{\left\|D D^{+}\right\|}\right) \\
& =\operatorname{tr}\left[C_{i}\left(V C_{j}\right) D\right] & & \left(\leq \sqrt{\left\|C_{i} C_{i}^{+}\right\|\left\|V C_{j}\right\| \sqrt{\left\|D D^{+}\right\|}}\right) \\
& =\operatorname{tr}\left[C_{i}\left(\left(V C_{j}\right) D\right)\right] & & \left(\leq\left\|C_{i}\right\|\left\|\left(V C_{j}\right) D\right\|\right)
\end{aligned}
$$

where $V=V_{I, J}$ and $D=D_{K, L}$ here are blocks of the Coulomb matrix and density matrix originating from the atomic pair $\langle I, J\rangle$ and $\langle K, L\rangle$, respectively. $C_{i}=C_{I i, K}^{I}$ is also a rank-2 tensor representing a sector of the triple expansion coefficients on atom pair $\langle I, K\rangle$ (i.e., $C_{I, K}$ introduced above) with fixed $\mathrm{AO}$ basis function $i$ (i.e., the rank-3 block tensor $C_{I, K}$ with fixed $i$ ). The crucial part in eq (17) is the different estimated upper bounds given in parenthesis for the same quantity. These different estimations can be utilized to filter out the insignificant elements at different stages of the calculation, all together leading to a highly efficient screening algorithm.

As mentioned above and explained in Appendix A.1, the best order of matrix multiplication to obtain $\mathcal{H}^{\mathrm{x}}$ is given by the last line of eq (17). The pseudocode of the accordingly designed screening algorithm for evaluating $\mathcal{H}^{\mathrm{X}}$ is illustrated in Algorithm 4. In this algorithm, the actual working procedure goes as follows. We first calculate and store all needed quantities $-\sqrt{\left\|C_{i} C_{i}^{+}\right\|},\left\|C_{i}\right\|, \sqrt{\left\|V V^{+}\right\|}$and $\sqrt{\left\|D D^{+}\right\|}$in advance. During the actual calculation process of $\mathcal{H}^{\mathrm{x}}$ matrices, one further evaluate quantities $\left\|V C_{j}\right\|$ and $\left\|\left(V C_{j}\right) D\right\|$. The three upper bounds listed in eq (17) are calculated at appropriate locations within the 
calculation loops, and compared to a pre-chosen threshold $\varepsilon_{\mathrm{CS}-\text { matrix }}$. If any of the three upper bounds is below the threshold, the corresponding elements of $\mathcal{H}^{\mathrm{x}}$ matrices are set to zero, without actually computing its value. In Sec. 4.1.2, we will present benchmark results regarding the influence of the threshold $\varepsilon_{\mathrm{CS}-\text { matrix }}$ on accuracy and computation cost.

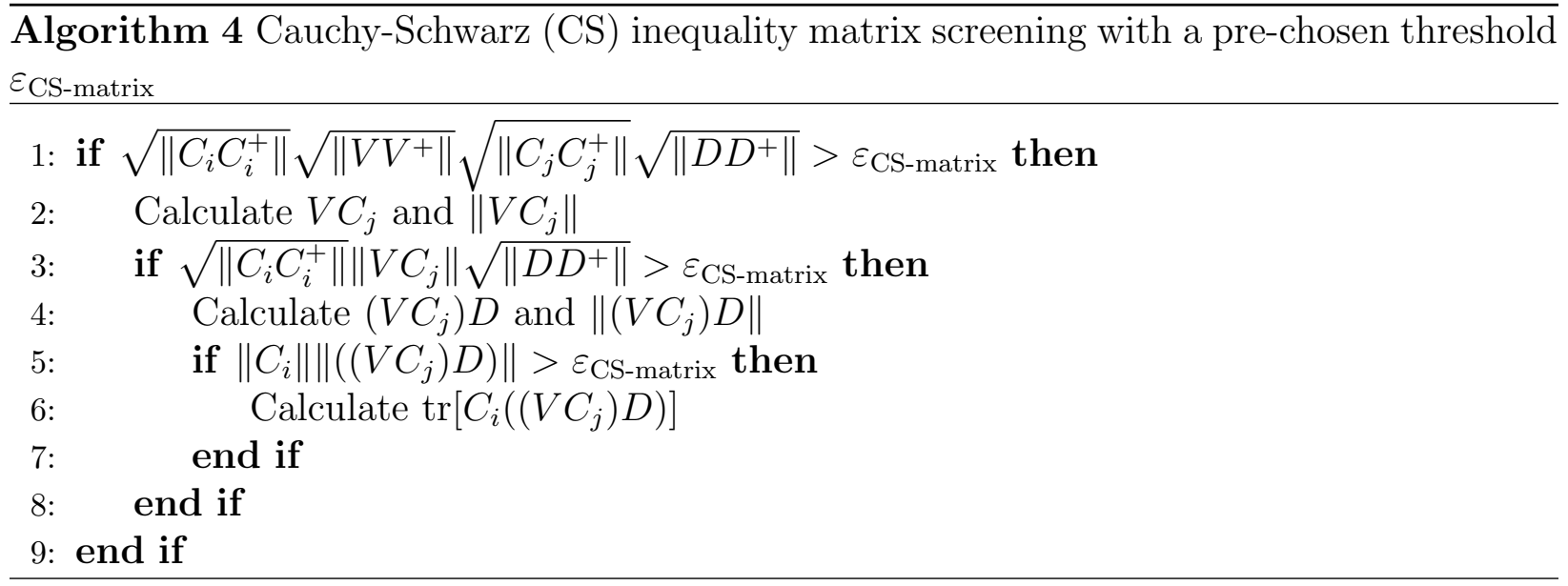

\subsubsection{Cauchy-Schwarz inequality ERI screening}

In addition to the screening criteria applied in Algorithm 4, one can apply one more criterion directly based on the full ERIs. The Cauchy-Schwarz inequality can again be applied to estimate the upper bound of each ERI quickly, ${ }^{93}$

$$
\left(\phi_{A a} \phi_{F f} \mid \phi_{B b} \phi_{G g}\right) \leq \sqrt{\left(\phi_{A a} \phi_{F f} \mid \phi_{A a} \phi_{F f}\right)} \sqrt{\left(\phi_{B b} \phi_{G g} \mid \phi_{B b} \phi_{G g}\right)} .
$$

In our implementation, we compute all the "diagonal" ERIs $\left(\phi_{A a} \phi_{F g} \mid \phi_{A a} \phi_{F g}\right)$ and determine

$$
\overline{\left(\phi_{A} \phi_{F} \mid \phi_{A} \phi_{F}\right)} \stackrel{\text { def }}{=} \max _{a \in A, f \in F}\left(\phi_{A a} \phi_{F f} \mid \phi_{A a} \phi_{F f}\right)
$$

beforehand, which can be done with relative ease since the number of these scales linearly with the system size. Now, the upper bound of each ERI can be found easily, and those below a given threshold $\varepsilon_{\text {CS-ERI }}$ can be disregarded without actually calculating them.

In ABACUS, we actually only calculate (implicitly) the partial ERIs $\left[\phi_{\underline{A} a} \phi_{F f} \mid \phi_{\underline{B} b} \phi_{G g}\right]$ 
and its three variant form. However, this does not affect the use of Cauchy-Schwarz inequality screening. Before calculating $\left[\phi_{\underline{A} a} \phi_{F f} \mid \phi_{\underline{B} b} \phi_{G g}\right]$ we first use (18) to check whether $\left(\phi_{A a} \phi_{F a} \mid \phi_{B b} \phi_{G g}\right)$ is needed or not. If not, then it's unnecessary to calculate $\left[\phi_{\underline{A} a} \phi_{F f} \mid \phi_{\underline{B} b} \phi_{G g}\right]$ and other three partial ERIs which form the full ERI (eq. (11)).

Combining the "matrix product screening" as discussed in Sec. 3.3.1 and the ERI-based screening discussed above, the entire flowchart of for evaluating the HFX matrix is presented in Algorithm 5. At a given atomic structure, the maximal "diagonal" ERI $\left(\phi_{A} \phi_{F} \mid \phi_{A} \phi_{F}\right)$ is first determined for each atomic pair. They are then used to filter out the atomic quartet whose ERIs are below a threshold $\varepsilon_{\mathrm{CS}-\mathrm{ERI}}$, at the beginning of the HFX calculation. The "matrix product screening" is only applied for those atomic sets $\{A, B, F, G\}$ which passed the ERI-based screening.

\subsection{Parallelization of the algorithm}

According to the algorithms described in Sec. 3.1, in particular Algorithm 2, our central parallelization strategy is to distribute the atom pairs $\langle A, B\rangle$ over different $\mathrm{CPU}$ cores. For each $\langle A, B\rangle$ pair, we search the neighbouring atoms $F$ and $G$ within certain cut-off radii, and calculate the corresponding $V_{A \alpha, B \beta}, C_{A a, F f}^{A \alpha}$ and $C_{B b, G g}^{B \beta}$ matrices. For a given atomic structure, these matrices are calculated once and stored in memory beforehand, since they depend only on the atomic structure and don't change during the self-consistent-field (SCF) cycles. The density matrices $D_{K k, L l}$ and HFX matrices $\Sigma_{I i, J j}^{\mathrm{X}}$, on the other hand, are updated at each iteration of the SCF loops. The full density matrix $D$ is calculated after diagonalizing the total Hamiltonian matrix $H$ in a $2 \mathrm{D}$ cyclic-block form, and hence initially also stored in the same distributed form as $H$. It is then redistributed over the $\langle A, B\rangle$ pairs via MPI communication tools, ${ }^{84}$ so that the needed matrix elements of $D$ are locally available when building the HFX via Algorithm 2. Once we have the needed $C_{A a, F f}^{A \alpha}$, $V_{A \alpha, B \beta}, C_{B b, G g}^{B \beta}$, and $D_{K k, L l}$ matrices ready in each individual MPI process, the $\mathcal{H}^{\mathrm{X}}$ matrices

can be calculated independently without any communication and the desired $\Sigma_{I i, J j}^{\mathrm{X}}$ matrix 


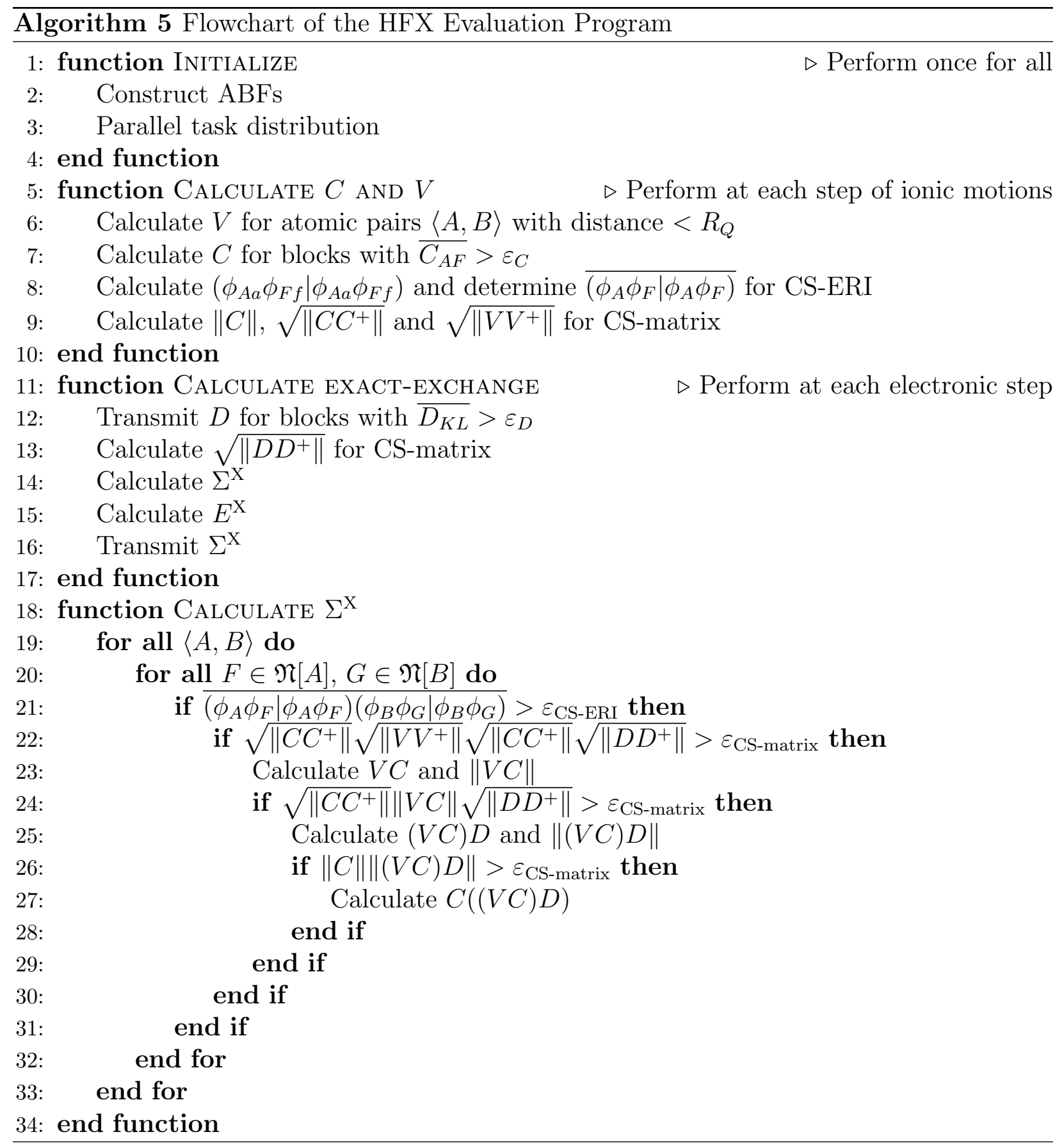


can be obtained from $\mathcal{H}^{\mathrm{x}}$ matrices (cf. Algorithm 2) via only light communications. After the locally distributed $\Sigma^{\mathrm{X}}$ (based on atomic pairs) is calculated, it will be transferred to the 2D cyclic-block form and added to the total Hamiltonian $H$. The major communication processes are illustrated in Fig. 2. The key feature of this parallelization algorithm is that only the relatively cheap $\Sigma^{\mathrm{X}}$ and $D$ matrices need to be redistributed and communicated among MPI processes, whereas the more expensive $C$ and $V$ matrices are evenly distributed over the MPI tasks and no data communications for these are needed.

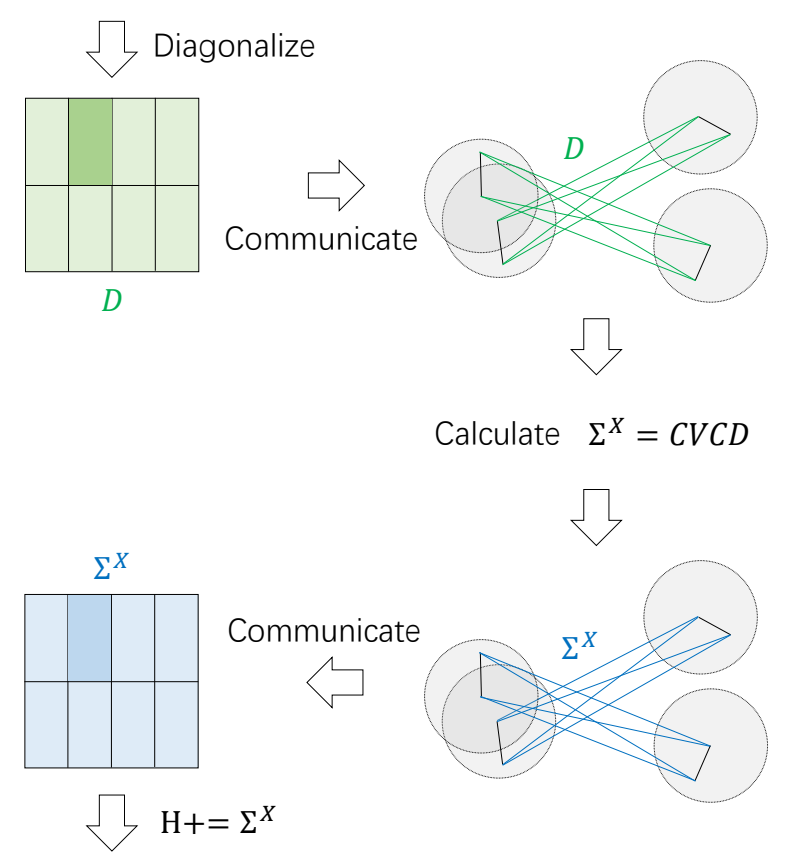

Figure 2: Sketch of the communication and data redistribution of the density matrix $D$ and HFX matrix $\Sigma^{\mathrm{X}}$. The left-hand side indicates the 2D cyclic-block form of the these two matrices, and the right-hand side indicates their local atom-pair based distribution form.

In principle, this part of calculations can be parallelized up to $M \times N_{\text {at }}$ CPU cores, where $N_{\text {at }}$ is the number of atoms in a single unit cell, and $M$ is the number of neighbouring atoms determined by the range of the $V$ matrix. The load balancing totally depends on the distribution of $\langle A, B\rangle$ pairs. A random distribution of the $\langle A, B\rangle$ pairs may cause severe load imbalance for wall time and computational bottleneck of memories, in particular for the non-uniform systems where the number of neighbouring atoms may vary greatly for different atoms. To overcome this difficulty, we developed two distribution schemes to improve the 
parallel efficiency. The first one is called "Machine-scheduling distribution", which is to improve the load balance based on computational time, and the second algorithm is called "K-means distribution", which can be used to reduce the memory usage. Depending on the availability of the computational resources, we choose one or the other algorithm to distribute the computational load to achieve optimal performance.

The parallelization of HFX calculations in ABACUS is a hybrid mode based on processes and threads. The MPI ${ }^{84}$ is adopted for parallelization over processes, while OpenMP ${ }^{94}$ is employed for parallelization over threads. The $\langle A, B\rangle$ pairs are distributed to processes according to distribution schemes discussed below, and then each process forks threads to claim and complete the distributed $\langle A, B\rangle$ pairs with dynamic schedule in OpenMP for load balance.

\subsubsection{Machine-scheduling distribution}

For each atomic pairs $\langle A, B\rangle$, the size of the corresponding $\mathcal{H}^{\mathrm{x}}(\sim C V C D)$ matrix is proportional to $|\mathfrak{N}[A]| *|\mathfrak{N}[B]|$, where $|\mathfrak{N}[A]|,|\mathfrak{N}[B]|$ are the numbers of neighbouring atoms of atom $A$ and $B$ respectively. We can think of the atoms in the system as the vertices of an undirected graph, whereas the atom pairs can be viewed as the edges. The computational cost of each edge $e=\langle A, B\rangle$ is roughly $N_{e}=|\mathfrak{N}[A]| *|\mathfrak{N}[B]|$, which is the weight of the edge. In this case, we try to distribute the weighted edges as evenly as possible among the CPU cores, to minimize the maximum computational load on one core. This becomes a classical machine scheduling problem. ${ }^{95}$ It's known that the global optimum solution of machine scheduling problem is NP-hard, ${ }^{96}$ and thus it is not possible to find the global optimum solution for large systems. However, one can find an approximate solution by the greedy

algorithm. ${ }^{97,98}$ It can be proven that the maximum computational cost on one core given by the approximate solution is not more than twice as that of the optimal solution. In practice, we find the greedy algorithm as presented in Algorithm 6 works very well. In Algorithm 6, $S_{p}$ denotes the list of tasks ("edges" in this case) in process $p$, whereas $W_{p}$ denote the com- 
putational loads ("weight") on process $p$. The meanings and relationships between $N_{e}, S_{p}$, $W_{p}$ are further graphically illustrated in Fig. 3.
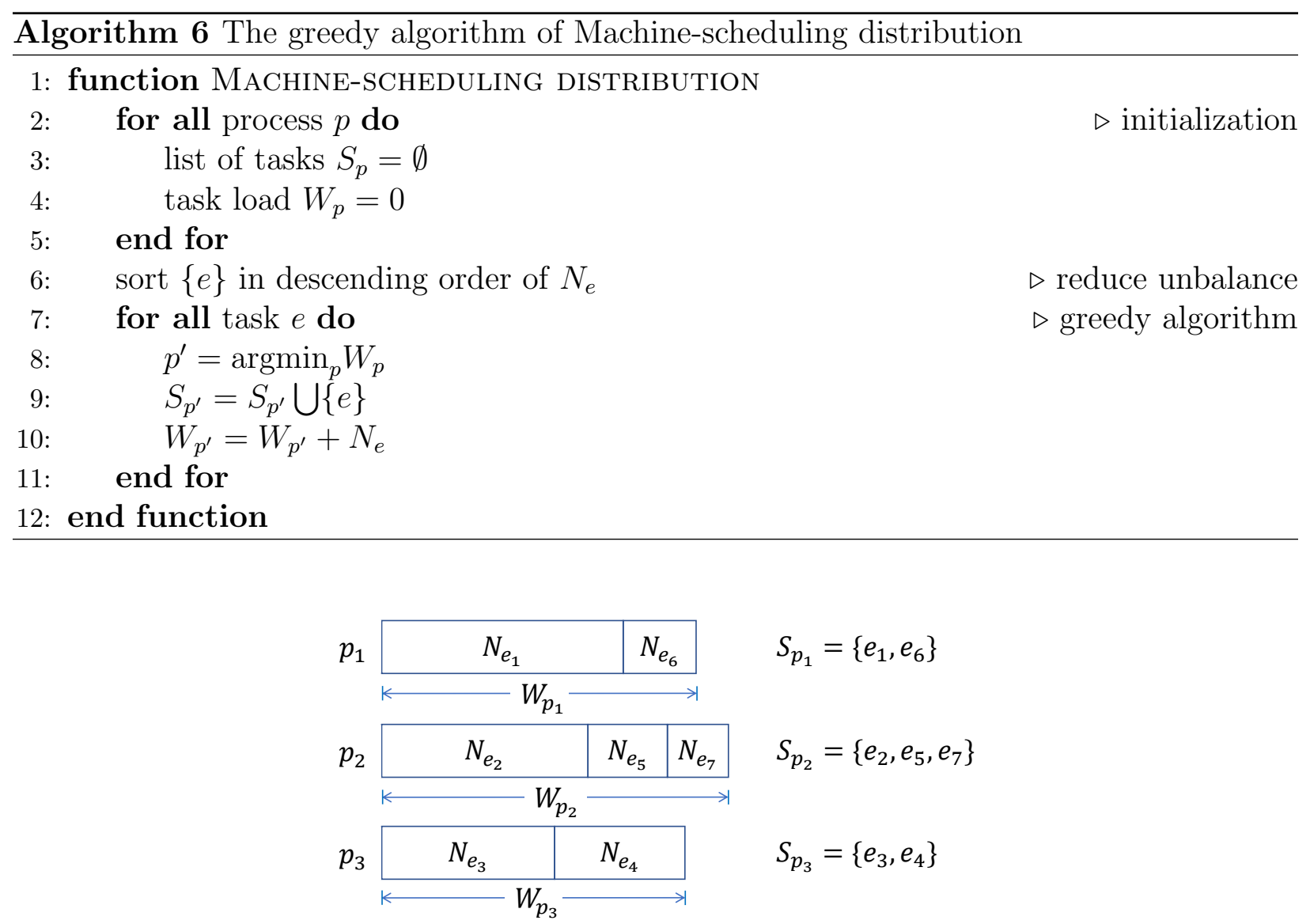

Figure 3: Graphical illustration of the process $p$, the list of tasks ("edges") $S_{p}$ on process $p$, the weight of a give task ("edge $e$ ") $N_{e}$, and the total weight of tasks $W_{p}$ on process $p$.

\subsubsection{K-means distribution}

The "Machine-scheduling distribution" algorithm discussed above is suitable for achieving load balance in computation time. However, it is not memory friendly. To understand this point, we first briefly analyze the memory consumption of the major arrays in parallel computation of the HFX matrix. The variation of the memory consumption upon increasing the number of compute nodes is a key factor that affects the scalability of the calculation. The sparsity of $C$ in large systems is guaranteed by the locality of NAOs, and its memory consumption increases linearly with system size. In our implementation, the memory footprint 
of $C$ in each compute node can be reduced by increasing the number of compute nodes. As for $V$, although it is dense for long-range Coulomb potential, the indices of the $V$ matrix are precisely those used for parallel distribution. Hence the memory storage of $V$ in all processes is non duplicated, and it memory consumption in each process is inversely proportional to the number of processes. On the other hand, the parallel distribution of $D$ and $\Sigma^{\mathrm{X}}$ is much more involved, and the memory consumption of these arrays may become the bottleneck for large size systems in parallel calculations. In practice $\Sigma^{\mathrm{X}}$ consumes more memory than $D$, and hence we take $\Sigma^{\mathrm{X}}$ as an example to analyze the problem. The the analysis of $\Sigma^{\mathrm{X}}$ applies to $D$ as well.

As illustrated in Algorithm 2, our actual implementation is based on the loop structure over atomic pairs $\langle A, B\rangle$. For each pair $\langle A, B\rangle$, one needs to evaluate contributions to four blocks of the HFX matrices, i.e., $\Sigma_{F f, G g}^{\mathrm{X}}, \Sigma_{F f, B b}^{\mathrm{X}}, \Sigma_{A a, G g}^{\mathrm{X}}$ and $\Sigma_{A a, B b}^{\mathrm{X}}$, among which $\Sigma_{F f, G g}^{\mathrm{X}}$ is most memory intensive because of the presence of different $F, G$ atoms in the neighborhood of $\langle A, B\rangle$ pair. A pictorial illustration of the situation is presented in Fig. 4. When a group of atom pairs $\langle A, B\rangle$ are distributed to one process $p$, the set of $\Sigma_{F f, G g}^{\mathrm{X}}$ that need to be stored in process $p$ is given by $\bigcup_{\langle A, B\rangle \in p} \bigcup_{F \in \mathfrak{N}[A]} \bigcup_{G \in \mathfrak{N}[B]} \Sigma_{F G}^{\mathrm{X}}$. This means that the memory consumption of $\Sigma_{F f, G g}^{\mathrm{X}}$ on a process $p$ is proportional to the union of the neighborhood regions of the atom $A$ 's and $B$ 's assigned to $p$, as illustrated in Fig. 4. To minimize the memory consumption of $\Sigma_{F f, G g}^{\mathrm{X}}$, the number of different $F(G)$ atoms in the neighborhood of $A(B)$ atoms distributed on the same process should be as few as possible. The "Machine-scheduling distribution" scheme doesn't consider the atom positions, and the $\langle A, B\rangle$ pairs and their neighbors in one process may spread all over the supercell. If this happens, simply increasing the number of compute nodes does not necessarily lead to the reduction of the memory consumption of $H$ on one process.

Based on the above analysis, it is obvious that minimizing the memory consumption for $\Sigma_{F f, G g}^{\mathrm{X}}$ on each process amounts to minimizing $\bigcup_{A} \mathfrak{N}[A]$, or equivalently, by maximizing the overlap of all $\mathfrak{N}[A]$ 's present in one process. Considering that the atomic cut-off radii of all 
chemical elements in the actual calculations are very close if not equal, this is essentially equivalent to making all $A$ 's allocated to each process as close as possible. The same principle applies to atom $B$.

Requiring the atom $A$ 's ( $B$ 's) allocated to the same process as close as possible is a typical clustering problem - an unsupervised learning problem in machine learning. Specifically, we need to sort the atoms into several groups, in the three-dimensional Euclidean space, with the aims that the atoms in each group are as close as possible. The K-means algorithm ${ }^{99}$ given in Algorithm 7 is chosen here to cluster atoms.

For short-range potential, such as HSE potential, etc., only $\langle A, B\rangle$ within certain ranges are needed, whereas for long-range potential, such as HF etc., almost all pairs of $\langle A, B\rangle$ are needed.

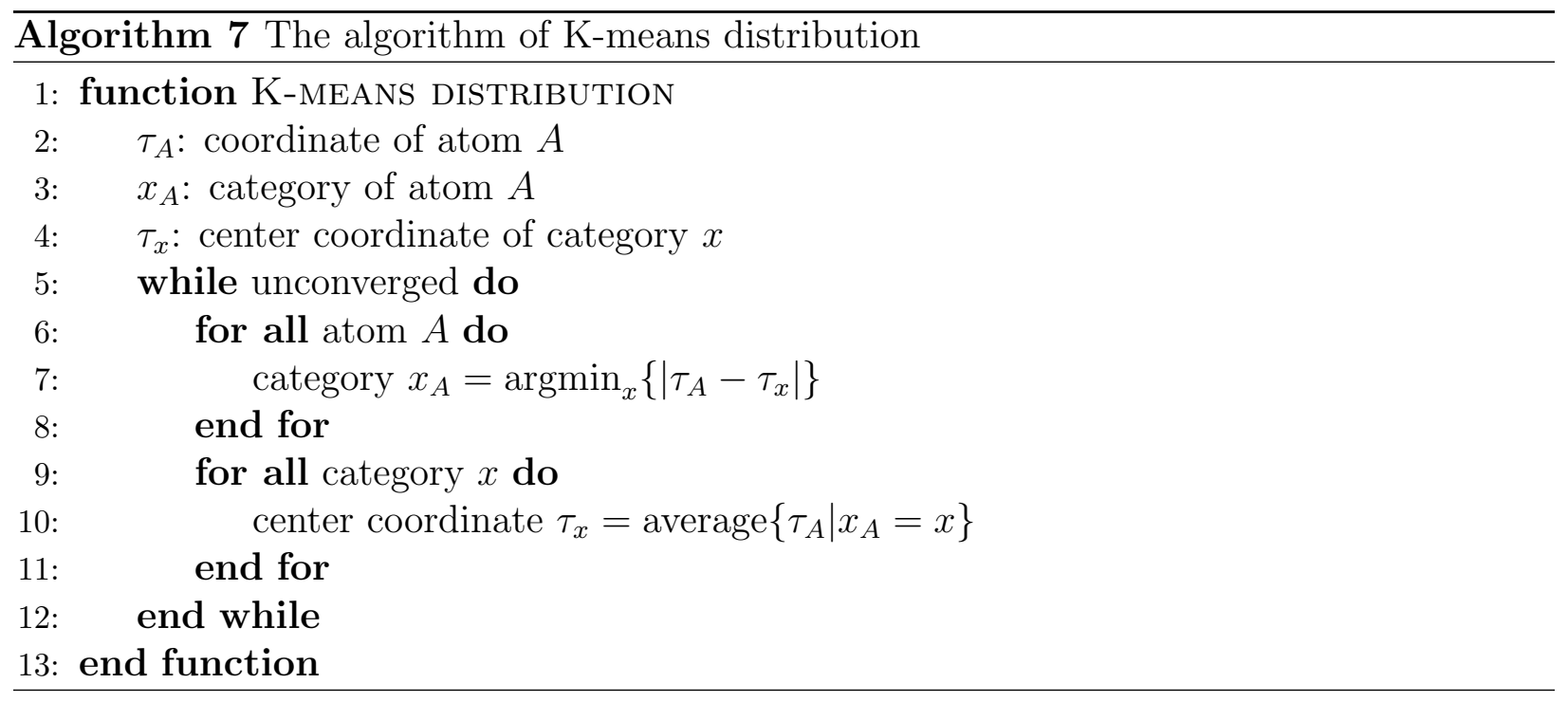

\section{Results and discussions}

In this section, we benchmark the efficiency and scalability of our algorithm and implementations as discussed in Secs. 2 and 3 via specific examples. We first demonstrate the effect of our screening techniques, which is the central part of our implementation (Sec 4.1). This is followed by an examination of the efficacy of the two parallel distribution schemes (Sec 4.2). 


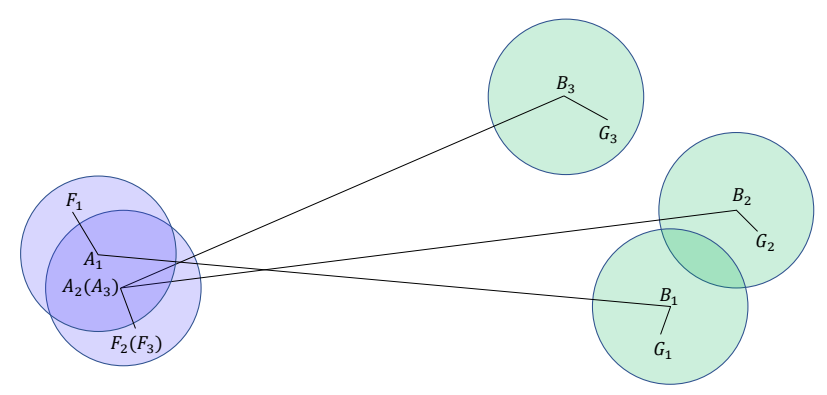

Figure 4: Illustration of the memory consumption of $\Sigma_{F f, G g}^{\mathrm{X}}$ on one process. For an atom pair $\langle A, B\rangle$, the set of $\Sigma_{F f, G g}^{\mathrm{X}}$ covers $\forall F \in \mathfrak{N}[A]$ and $\forall G \in \mathfrak{N}[B]$. For a group of atom pairs $\{\langle A, B\rangle\}$ distributed to one process, the set of $\Sigma_{F f, G g}^{\mathrm{X}}$ is the union of all $F, G$ atoms in the neighborhood of all these $\{\langle A, B\rangle\}$ atom pairs.

A comprehensive benchmark study of the overall performance of our HFX implementation with respect to the system size and the number of CPU cores is presented in Sec. 4.3. Finally the advantage of our implementation for band structure calculations are discussed in Sec. 4.4. Note that we are concentrating here the efficiency aspect of our implementation, and the accuracy aspect has been reported in a previous publication. ${ }^{58}$

\subsection{Effects of screening}

\subsubsection{Pre-screening of individual $C, V$ and $D$ matrices}

We perform test calculations on a unit cell of $N_{\text {at }}$ atoms with $N_{k x} \times N_{k y} \times N_{k z}$ k-points, which corresponds to a supercell of $N_{\text {at }} \times N_{k x} \times N_{k y} \times N_{k z}$ atoms with a single k-point. In this setting, the atom $A$ can be restricted within the central unit cell, whereas the atoms $B, F$, $G$ run over the entire supercell. Thus, all the $C, V$, and $D$ matrices have the same real-space data structure and hence the same sparsity as if we are dealing with a $\Gamma$-only supercell with $N_{\text {at }} \times N_{k x} \times N_{k y} \times N_{k z}$ atoms. Therefore, a small unit cell with a dense $k$-point mesh is well suitable for testing the effect brought about by screening out the insignificant elements of $C, V$ and $D$ matrices. Yet, compared to directly dealing with a large supercell, the computational cost can be significantly reduced, thanks to the translational symmetry.

As a specific test example, we performed HSE06 ${ }^{100}$ calculations (with the screening pa- 
rameter $\mu=0.11$ ) for $\mathrm{Si}$ crystal. The lattice constant is chosen to be 10.236 Bohr, and a $8 \times 8 \times 8 \mathbf{k}$-point mesh is used for Brillouin zone (BZ) integration. The energy cut-off for determining the uniform real-space integration grid for charge density is set to be 240 Ry. We use a NAO DZP basis $[2 s 2 p 1 d]$ for the one-electron basis set; for ABFs, an optimized $[5 s 4 p 3 d]$ set of orbitals ${ }^{58}$ is used. The cut-off radii of both NAOs and ABFs are set to 8 Bohr.

Now we first check what happens if we pre-screen the small matrix elements of individual $C, V$, and $D$ matrices, i.e., the influence of the pre-screening on the numerical accuracy, the computation time, and the memory consumption. Here, the numerical accuracy of the calculations is measured by the error of the obtained band gap, given by $\delta E_{g}=\left|E_{g}^{\mathrm{scr}}-E_{g}^{\mathrm{ref}}\right|$ where $E_{g}^{\text {scr }}$ is the bang gap value obtained with screening and $E_{g}^{\text {ref }}$ is the reference value obtained without applying screening - by setting the thresholding parameters to zero, or in case of the $V$ matrix, by setting $R_{Q}$ to a big value. Here the error of the band gap is used as an measurement of the computational accuracy, as it is more sensitive to screening thresholds, compared to the variational quantities such as the ground-state total energy. Fig. 5 presents the band gap errors, memory consumption for $C, V, D$ matrices, and the computation time of evaluating the HFX matrix in one SCF iteration as a function of their respective screening thresholds or cut-off parameters. The calculation was performed on one Intel(R) Xeon(R) CPU (E5-2640 v2 @ 2.00GHz) core. As discussed in Sec. 3.2, $\varepsilon_{C}$ and $\varepsilon_{D}$ are used to directly filter the subblocks of $C$ and $D$ matrices, and a smaller values of $\varepsilon_{C}$ and $\varepsilon_{D}$ means more accurate calculations. The pre-screening of the $V$ matrix, on the other hand, is controlled by a cut-off radius $R_{Q}$ of the Coulomb potential $Q(r)$ associated with the ABFs, as introduced in Sec. 3.2.2. Obviously, a larger $R_{Q}$ corresponds to smaller numerical errors.

The test calculations are done in a successive way. Namely, we first examine the influence of $R_{Q}$, while setting $\varepsilon_{C}$ and $\varepsilon_{D}$ to be zero. Then, with a fixed value of $R_{Q}$ and zero $\varepsilon_{D}$ value, we look at the impact of $\varepsilon_{C}$. Finally, with fixed $R_{Q}$ and a finite $\varepsilon_{C}$ threshold, we check the 

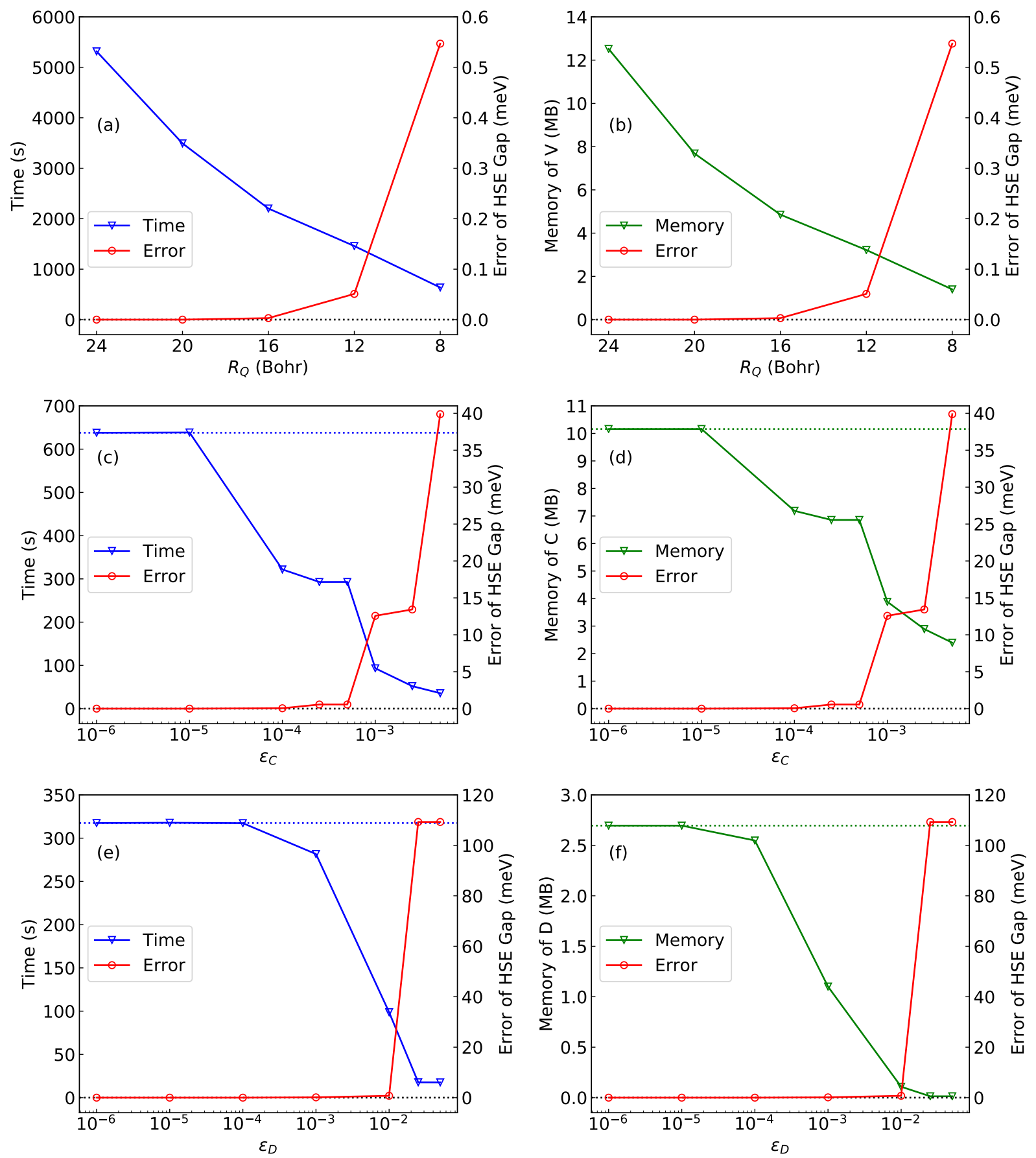

Figure 5: Computation times (left panels) and memory footprints (right panels) as a function of the cut-off/thresholding parameters in pre-screening the individual $V$ (upper panels), $C$ (middle panels), and $D$ (bottom panels) matrix. In all panels, the accompanying band gap errors are plotted as an indicator of the numerical accuracy of the calculations. 
effect of $\varepsilon_{D}$. Inspection of Fig. 5 reveals that, for all three matrices, there exists a window of parameter values, within which there is almost no loss of accuracy (i.e., the obtained band gap staying essentially unchanged), yet the computation time and memory consumption is significantly reduced. In the HSE06 functional, the $Q(\mathbf{r})$ itself is short-ranged, which allows for introducing a small cut-off radius for $Q(\mathbf{r})$ to screen out the insignificant elements of the $V$ matrix. As shown in Fig. 5(a) and (b), when the cut-off radius $R_{Q}$ is reduced from 24 Bohr to 8 Bohr, the incurred band gap error is negligible (less than $1 \mathrm{meV}$ ), whereas the computation time is reduced from $5317 \mathrm{~s}$ to $637 \mathrm{~s}$ and the memory cost reduced from 12.5 MB to 1.4 MB. Such a drastic reduction of the computation time and memory consumption certainly benefits from the short-ranged nature of the $V$ matrix in screened HDFs such as HSE. In contrast, if large-range part of the Coulomb matrix is needed, such as in HartreeFock, or long-range-corrected HDFs, a rather large $R_{Q}(\sim 50$ Bohr $)$ is needed, and the memory storage of the $V$ matrix, as well as the computation time, will get significantly more expensive.

Fig. 5(a) and (b) show the change of the computation time and the memory cost of the $C$ matrix as a function of $\varepsilon_{C}$, with $R_{Q}=8 \mathrm{Bohr}$ and $\varepsilon_{D}=0$. It can be seen that, if $\varepsilon_{C}$ is set to $1 \times 10^{-4}$, compared to $\varepsilon_{C}=0$ (no pre-screening) the computing time is reduced from $637 \mathrm{~s}$ to $322 \mathrm{~s}$ (nearly $50 \%$ reduction) and the memory of the $C$ matrix from $10.2 \mathrm{MB}$ to 7.2 MB (nearly 30\% reduction), while the computed band gap is barely affected. Note that the zero elements in $C$ due to the finite cut-off radius of the AOs have already been excluded at the very beginning the calculation, and the memory reduction recorded here is due to the small but finite matrix elements of $C$. When increasing $\varepsilon_{C}$ from $5 \times 10^{-4}$ to $2.5 \times 10^{-3}$, the computing time goes further down to 52 s (i.e., more than $90 \%$ reduction) and the memory cost of $C$ matrices down to $2.9 \mathrm{MB}$ (i.e., more than $70 \%$ reduction), but now a visible band gap error of $13.4 \mathrm{meV}$ is incurred. However, further increasing $\varepsilon_{C}$ beyond $10^{-3}$ leads to a rapid increase of the band gap error, which should be avoided.

Finally we check the influence of $\varepsilon_{D}$ with fixed $R_{Q}=8$ Bohr and $\varepsilon_{C}=10^{-4}$, and the 
obtained results are reported in Fig. 5(e) and (f). One can see that, when increasing $\varepsilon_{D}$ from 0 to $10^{-3}$, the computation time is reduced from $322 \mathrm{~s}$ to $282 \mathrm{~s}$, and the memory cost of $D$ is reduced from 2.7 MB to 1.1 MB. When further increasing $\varepsilon_{D}$ to $10^{-2}$, the computation time is drastically reduced from $282 \mathrm{~s}$ to $99 \mathrm{~s}$, and the memory consumption from $1.1 \mathrm{MB}$ to 0.1 MB. In the mean time, no noticeable change of the band gap is observed. Such a significant saving in computation time and memory storage is enabled the fact that silicon crystal is an insulator, and its density matrix decays exponentially in real space. However, if increasing $\varepsilon_{D}$ even further a little bit (say, to $2.5 \times 10^{-2}$ ), a rapid increase of the band gap error to $\sim$ $0.1 \mathrm{eV}$ occurs. Therefore, one needs to be very cautious when choosing the $\varepsilon_{D}$ parameter. In practice, we found that a conservative value of $10^{-3}$ is safe and hence is recommended in practical calculations.

\subsubsection{Screening based on Cauchy-Schwarz inequalities}

After pre-screening individual $C, V$, and $D$ matrices, we can further apply the screening techniques based on Cauchy-Schwarz inequalities, as discussed in Sec. 3.3.2 and Sec. 3.3.1, to filter out those matrix elements that jointly lead to negligibly small $\mathcal{H}^{\mathrm{X}}$ matrix elements. Any remaining insignificant elements of $C, V$, and $D$ matrices that passed the initial prescreening step, will be identified and further excluded here.

According to the screening workflow outlined in Algorithm 5, we first apply the ERIbased Cauchy-Schwarz screening procedure and then the "matrix-product" based screening one. Note that, at this point, the pre-screening of individual $C, V$, and $D$ have already been performed with $R_{Q}=8$ Bohr, $\varepsilon_{C}=10^{-4}$, and $\varepsilon_{D}=10^{-3}$. Now, the memory storage

of the $\Sigma^{\mathrm{X}}$ matrix is used to measure the effect on the memory consumption due to the Cauchy-Schwarz inequality screenings. Fig. 6(a) and (b) present the computation time and

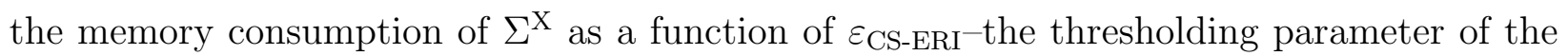
ERI-based Cauchy-Schwarz inequality ERI screening. When setting $\varepsilon_{\text {CS-ERI }}=10^{-3}$, the induced error of the band gap is about $0.9 \mathrm{eV}$, while the computation time is reduced from 
$282 \mathrm{~s}$ to $171 \mathrm{~s}$. In this case, the influence on the memory cost of $\Sigma^{\mathrm{X}}$ is minor. Further increasing $\varepsilon_{\text {CS-ERI }}$ to $10^{-2}$ can accelerate the calculation by another a factor of 3 , but the incurred error rises to about $20 \mathrm{meV}$, which is not recommended.

A final step is the Cauchy-Schwarz inequality matrix screening as outlined in Algorithm 4. The computation time, the memory consumption of $\Sigma^{\mathrm{X}}$, and the band gap error as a function of the truncation threshold $\varepsilon_{\text {CS-matrix }}$ is shown in Fig. 6(c) and (d). As $\varepsilon_{\text {CS-matrix }}$ increases from 0 to $10^{-6}$, the computation time for evaluating HFX matrix is reduced from $172 \mathrm{~s}$ to $127 \mathrm{~s}$ and the memory cost of $\Sigma^{\mathrm{X}}$ decreases from 2.6 MB to $1.3 \mathrm{MB}$. In the meantime, the accompanying band gap error is only $0.77 \mathrm{meV}$. Further increasing $\varepsilon_{\mathrm{CS}-\mathrm{matrix}}$ from $10^{-6}$ to $10^{-5}$, the computation time is reduced to $82 \mathrm{~s}$ and memory of $\Sigma^{\mathrm{X}}$ to $0.9 \mathrm{MB}$, but the band gap error is increased to $11 \mathrm{meV}$.

In summary, by applying both the pre-screening of individual matrices and the screening procedures based on Cauchy-Schwarz inequalities, the computation time for evaluating the HFX matrix in one iteration is reduced by a factor of 40 , whereas the total memory consumption of the four most memory intensive matrices $-C, V, D, \Sigma-$ is reduced by a factor of 3. The accumulated error of the band gap, compared to the reference value without applying any screening, is only $0.57 \mathrm{meV}$. Similarly, the incurred error in the absolute HSE06 total energy, which was not reported in the above analysis, is only $0.20 \mathrm{meV}$. (Note that the errors induced in individual steps might compensate each other, but are always in the same order of magnitude.) Obviously, if one can tolerate bigger errors, say $10 \mathrm{meV}$ in band gap, the savings in computation time and memory storage will be even more significant.

\subsection{Parallel distribution schemes}

\subsubsection{Machine-scheduling distribution}

In parallel computing, keeping good load balance among the processes is a key requirement to enable massively parallel calculations. In our implementation, this is achieved by the so-called "Machine-scheduling distribution", as described in Sec. 3.4.1. To assess the per- 

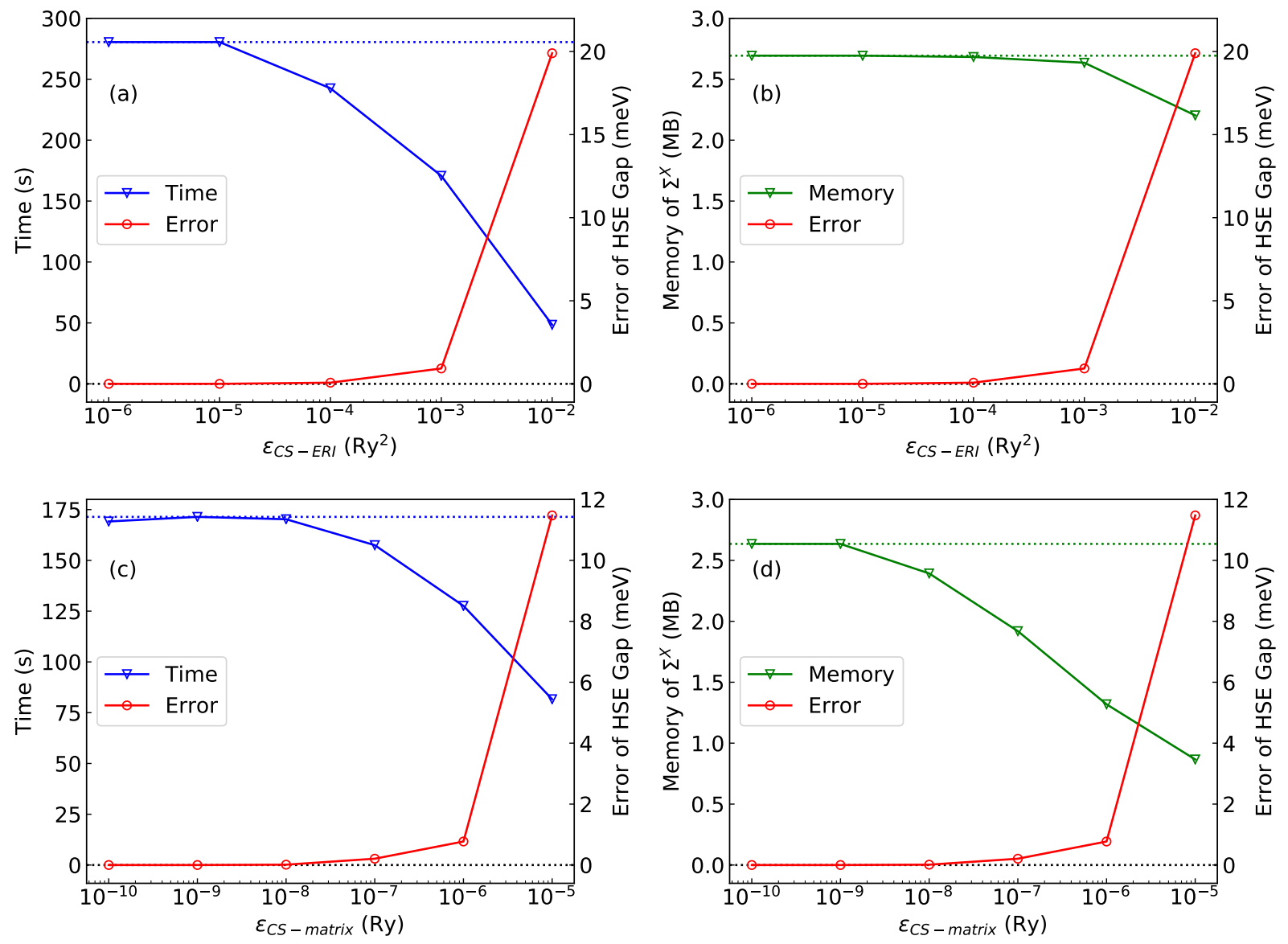

Figure 6: Computation times (left panels) and memory footprints of $\Sigma^{\mathrm{X}}$ (right panels) as a function of the thresholding parameters of the ERI-based (upper panels) and "matrix product" based (lower panels) Cauchy-Schwarz screening. In all panels, the induced band gap errors are plotted as an indicator of the numerical accuracy of the calculations. 
formance of this distribution scheme, we compare it with a straightforward parallelization scheme where the atom pairs are distributed randomly, only requiring that the numbers of atom pairs assigned to each process are equal.

The test system chosen here is a DNA fragment containing 788 atoms, composed of 12 AT basis pairs, as studied in Ref. 101. The load balance is measured by the ratio between the maximal time consumed on one process $\left(\Delta t_{\max }\right)$ and the average time over all processes $\left(\Delta t_{\mathrm{av}}\right)$. If the load balancing is perfect, $\Delta t_{\max } / \Delta t_{\mathrm{av}}$ ratio should equal 1; otherwise this ratio will be larger than 1. Obviously, the larger the ratio is, the worse the load balance. We note that the global communication time between different processes is accounted for here, as it is the "common" time shared by all processes.

As a numerical experiment, we carried out six independent HSE06 calculations, respectively, on 1, 2, 4, 8, 16, and 32 compute nodes, each with $24 \mathrm{CPU}$ cores. Consistent with the computer architecture, the calculations are parallelized using 1, 2, 4, 8, 16, and $32 \mathrm{MPI}$ processes, each process consisting of 24 threads. When running on one compute node (1 process $\times 24$ threads), both "Machine-scheduling distribution" and "random distribution" schemes perform perfectly, with $\Delta t_{\max } / \Delta t_{\mathrm{av}}$ ratio being essentially one, as it should be. As the number of processes increases, the $\Delta t_{\max } / \Delta t_{\mathrm{av}}$ ratio of the "random distribution" scheme grows gradually, reaching 2.2 when 32 nodes (768 CPU cores) are used, meaning that the maximum computing time on one process is more than twice of the average computing time. In contrast, the $\Delta t_{\max } / \Delta t_{\mathrm{av}}$ ratio of the "Machine-scheduling distribution" scheme stays very close to 1 , increasing only slightly when more CPU cores are used. With 32 processes (768 CPU cores), the wall time is still less than 1.2 times of the ideal time. These results clearly demonstrate that, with the "Machine-scheduling distribution" scheme, one can achieve excellent load balancing in parallel computing even for very inhomogeneous systems. 


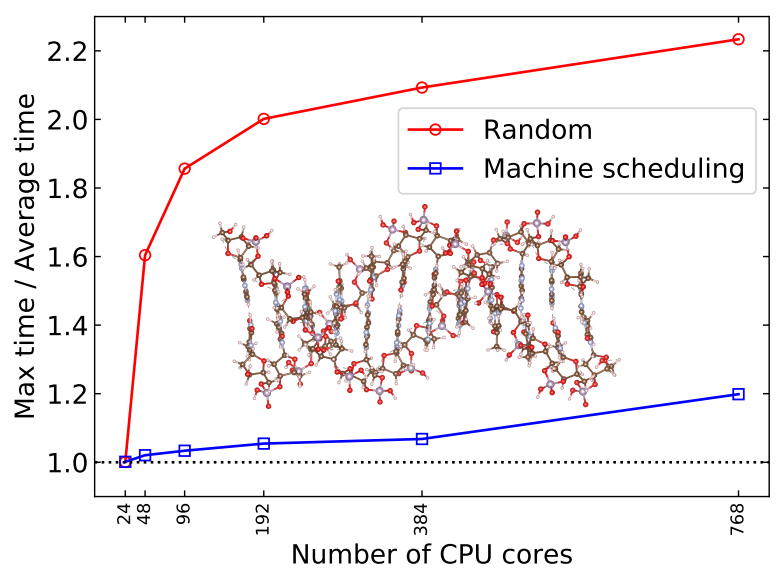

Figure 7: The "Max time/Average time" $\left(\Delta t_{\max } / \Delta t_{\mathrm{av}}\right)$ ratio for the "random distribution" (red circles) and "Machine-scheduling distribution" (blue squares) as a function of the number of CPU cores. Test calculations are done for a DNA fragment.

\subsubsection{K-means distribution}

As discussed in Sec. 3.4.2, in addition to the "Machine-scheduling distribution" scheme intended to improve the load balance of computation time, we offer an alternative, the so-called "K-means distribution" scheme to reduce the memory footprints. In our implementation, the memory consumption scales roughly linearly with the size of the unit cell, and can become a bottleneck for very big supercells. In these cases, the "K-means distribution" scheme enables calculations that would otherwise not run.

To test the performance of the "K-means distribution" scheme, we carried out a series of T-only HSE06 calculations for Si crystal with different unit cell sizes and increasing number of MPI processes. The maximal memory footprints for the $\Sigma^{\mathrm{X}}$ matrix per process for both "K-means distribution" and "random distribution" schemes are presented in Fig. 8 as a function of the number of MPI process (again each process running on $24 \mathrm{CPU}$ cores with shared memory) Different curves in Fig. 8 correspond to different sizes of the unit cell, containing 64, 128, 256, 512, and 1024 Si atoms, respectively. The computation parameters (basis sets, cut-off energy, and cut-off radii) are the same as those used in Sec. 4.1.1. The screening thresholds (or truncation parameters) are chosen to be $R_{Q}=8$ Bohr, $\varepsilon_{C}=10^{-3}$, 
$\varepsilon_{D}=10^{-2}, \varepsilon_{\mathrm{CS}-\mathrm{ERI}}=10^{-3}, \varepsilon_{\mathrm{CS} \text {-matrix }}=10^{-5}$. Tests showed that the incurred band gap error with these screening parameter settings is below $10 \mathrm{meV}$.

As discussed in Sec. 3.4.2, in our current parallelization algorithm, the same sublocks of the $\Sigma^{\mathrm{X}}$ matrix have to be stored in different processes, leading to a duplication of the memory storage of the $\Sigma^{\mathrm{X}}$ matrix. This may become a bottleneck for large-scale calculations. Fig. 8 clearly demonstrates the supremacy of the "K-means distribution" scheme (solid lines) over the unoptimzed "random distribution scheme" (dashed lines) in reducing the memory cost as the number of processes increases. With two processes, the "K-means distribution" scheme gains a factor of 1.25 memory saving, while this number steadily increases to 4.7 for 64 processes. Thus, the "K-means distribution" scheme can be invoked when there is a lack of memory, in particular in cases of massively parallel calculations.

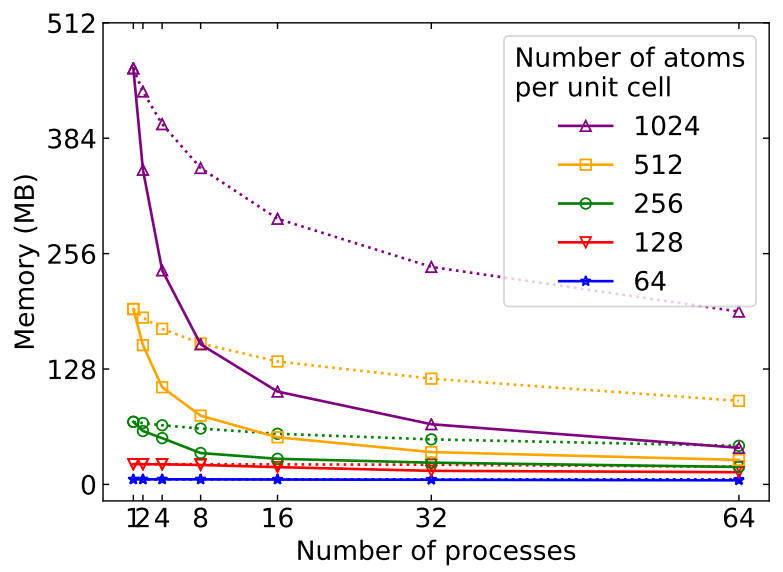

Figure 8: The maximal memory footprint of $\Sigma^{\mathrm{X}}$ among all runtime processes as a function of the number of MPI processes, for different unit cell sizes. The solid lines correspond to the "K-means distribution" scheme whereas the dashed lines represent the "random distribution" scheme.

\subsection{Overall performance}

To document the scaling behavior of the computational time for building the HFX matrix with the increase of system size and computing resource, we performed $\Gamma$-only HSE06 calculations for Si crystal with different supercell size and using different number of CPU cores. 


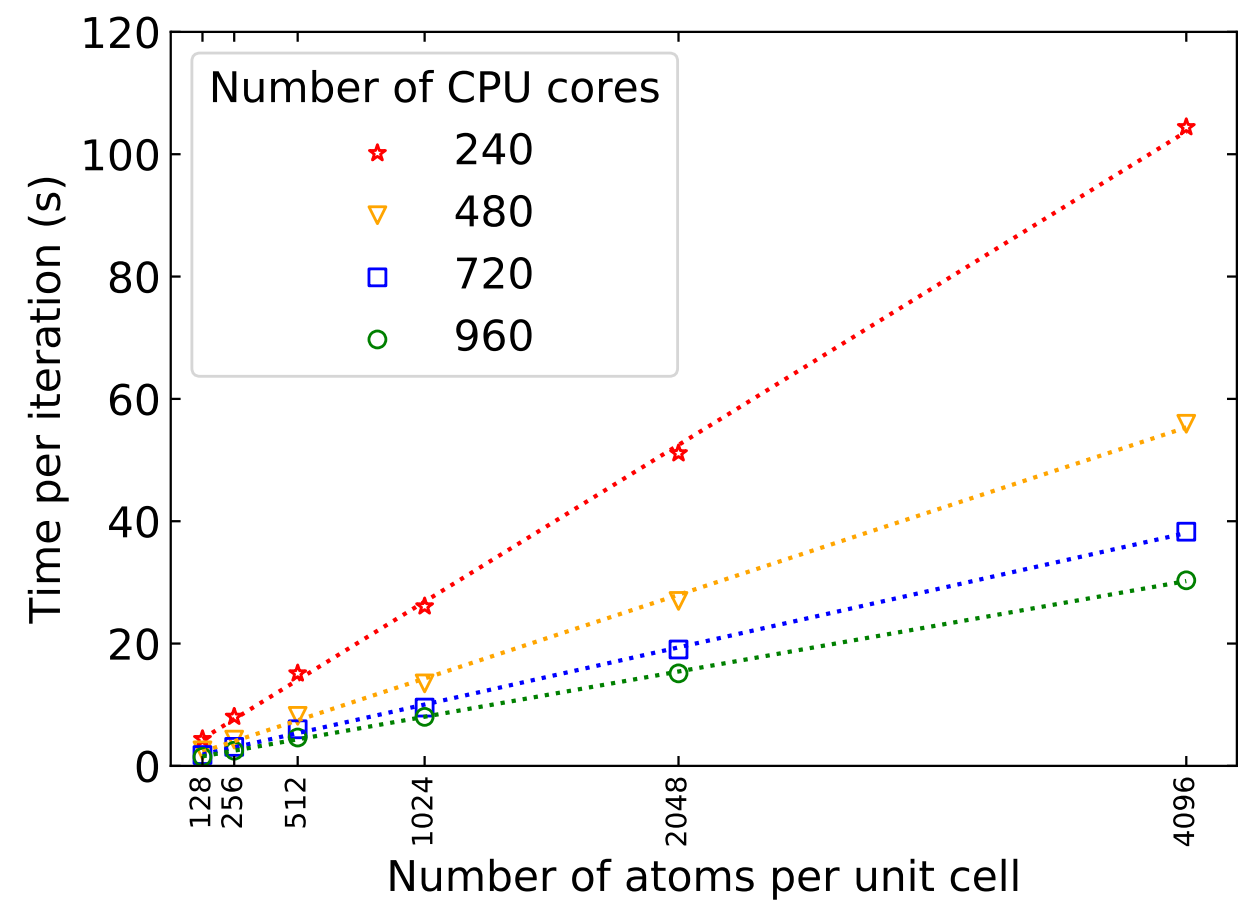

Figure 9: Computation time for building the HFX matrix per SCF iteration as a function of the system size for different amount of CPU resources. Test systems are Si crystal with various supercell sizes. 
The computation time recorded here is the wall time per iteration of evaluating and transmitting the $\Sigma^{\mathrm{X}}$ matrix, which is the most time-consuming part of the entire HDF calculation for system sizes tested so far (4906 atoms per unit cell). The basic computation parameters and screening thresholds are identical to those used in Sec. 4.2.2. Since there is no memory shortage problem in these calculations, the "Machine-scheduling distribution" scheme is used to achieve good load balances. All calculations are done on the Tianhe-2 supercomputer, where each node has two Intel(R) Xeon(R) CPUs (E5-2692 v2 @ 2.20GHz).

Fig. 9 presents the wall times as a function of the system size, i.e., the number of atoms in the supercell, for different numbers of CPU cores. We have tested from the smallest system containing $128 \mathrm{Si}$ atoms to the largest system containing $4096 \mathrm{Si}$ atoms. The size of the systems we have looked at ranges from 128 atoms up to 4096 atoms in the supercell. The calculations employs 10, 20, 30 and 40 processes $\times 24$ threads, running on 240, 480, 720 and 960 CPU cores, respectively. As can be seen from Fig. 9, in all parallel runs with different processes, the wall time scales almost linearly with the number of atoms in the calculations. A linear fit of the data obtained using $960 \mathrm{CPU}$ cores yields $t=0.0072 N_{\mathrm{at}}+0.6401$ (coefficient of determination $\left.R^{2}=0.9997\right)$. Although the linear scaling behavior is expected from the underlying algorithms, this benchmark test proves the efficacy of our implementation in ABACUS. Furthermore, the absolute timings presented in Fig. 9 indicate the prefactor of our linear-scaling algorithm is rather small - a feature that is vitally important for the usability of the code for practical calculations.

In Fig. 10, the wall times of the calculations presented above are re-plotted as a function of (the inverse of) the number of CPU cores. It can be seen that, for all system sizes, the computation time decreases linearly as the number of CPU cores increases. Even for the smallest system containing only $128 \mathrm{Si}$ atoms, the linear scaling is still perfect up to 960 CPU cores. For the 4096-atom system - the largest one tested in this work, the calculation takes about $104 \mathrm{~s}$ for one HFX evaluation step using $240 \mathrm{CPU}$ cores, and the computation time is reduced to about $30 \mathrm{~s}$, if $960 \mathrm{CPU}$ cores are used. A linear fit for the data of the 


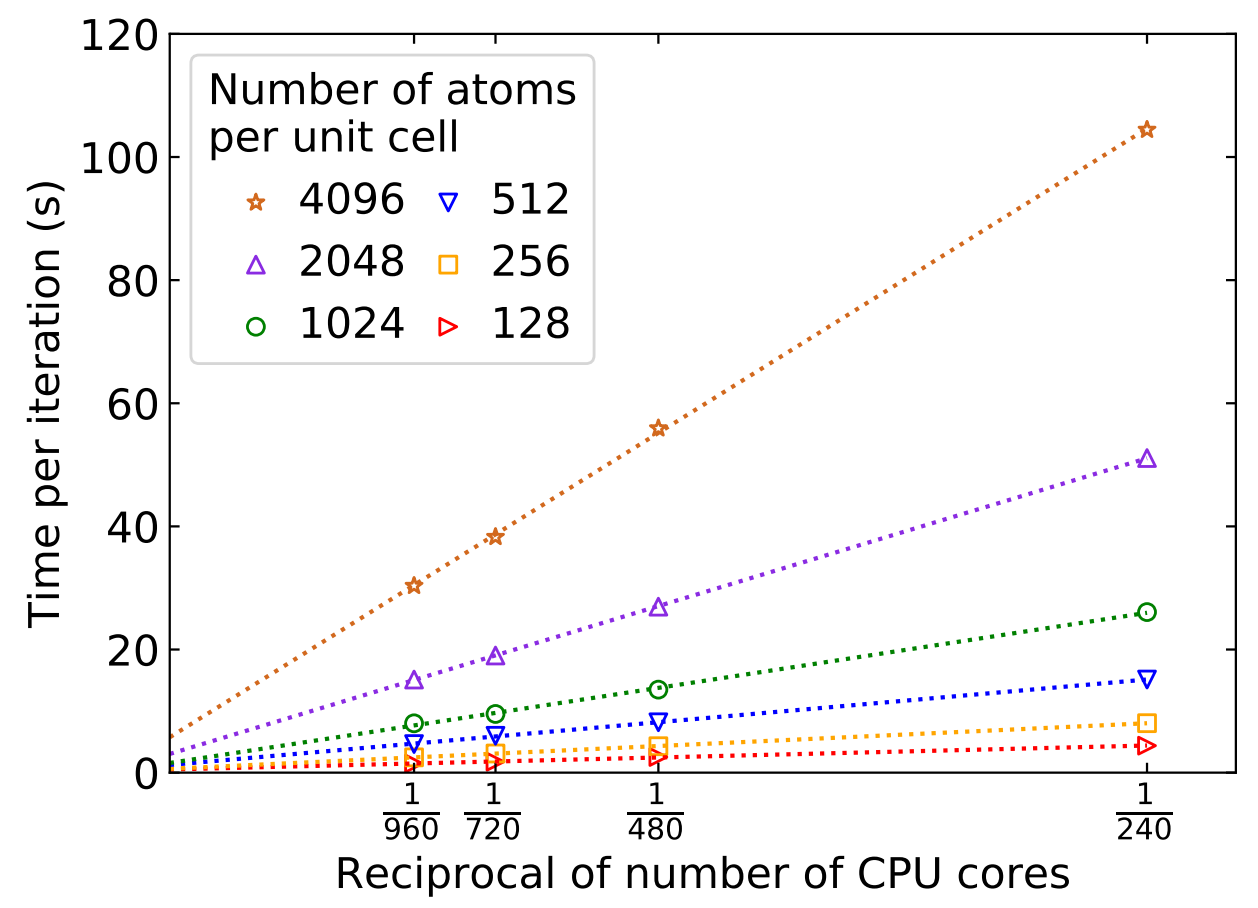

Figure 10: Computation time for building the HFX matrix per SCF iteration as a function of the number of CPU cores for different supercell sizes. Test systems are Si crystal with various supercell sizes. 
4096-atom system yields $t=23731 N_{\text {core }}^{-1}+5.7447\left(R^{2}=0.9998\right)$. The small prefactor in our linear-scaling implementation with respect to the system size and the excellent parallel efficiency allows to tackle large-scale systems with relative ease. With our implementation in ABACUS, HSE06 calculations for systems with a few thousands of atoms can be routinely performed. In fact, given the linear-scaling behavior of our implementation with respect to the system size and the number of CPU cores, there is in principle to handle systems with tens of thousands of atoms. However, in that case, diagonalizing the Hamiltonian matrix will become the new bottleneck - a challenge that is common to all KS or gKS-DFT calculations.

\subsection{Band structure calculations}

One of the additional advantage of the present implementation is that the electronic band structures of HDF calculations can be easily obtained. We briefly discuss this point in this subsection. In our implementation, we first obtain the HFX matrix in real space $-\Sigma_{I i, J j}^{\mathrm{X}}$, as indicated in eq (2), and then Fourier-transform it to $\mathbf{k}$ space. Note that in our notational system, the atom $I$, and $J$ can be located in different unit cells $\mathbf{R}_{I}$ and $\mathbf{R}_{J}$, and thus can be rewritten as

$$
\sum_{I i, J j}^{\mathrm{X}}=\sum_{\tilde{I}\left(\mathbf{R}_{I}\right) i, \tilde{J}\left(\mathbf{R}_{J}\right) j}^{\mathrm{X}}=\sum_{\tilde{I} i, \tilde{J} j}^{\mathrm{X}}\left(\mathbf{R}_{J}-\mathbf{R}_{I}\right)=\sum_{\tilde{I}, \tilde{J} j}^{\mathrm{X}}(\mathbf{R})
$$

where $\tilde{I}$, and $\tilde{J}$ denote the atomic indices in one unit cell and $\mathbf{R}=\mathbf{R}_{J}-\mathbf{R}_{I}$. For most systems, the exchange interactions are short-ranged, meaning that the matrix elements of $\sum_{\tilde{I}, \tilde{J} j}^{\mathrm{X} j}(\mathbf{R})$ are vanishingly small for $|\mathbf{R}|>R_{\max }$ where $R_{\max }$ is certain critical length.

After self-consistent HDF calculations, we can obtain the real-space HFX matrix $\Sigma_{\tilde{I}, \tilde{J} j}^{\mathrm{X}}(\mathbf{R})$ for all lattice vectors with $|\mathbf{R}|<=R_{\max }$, and merge it with the local part of the gKS Hamiltonian to get the full Hamiltonian. Once the the full gKS Hamiltonian $H_{\tilde{I}, \tilde{J} j}(\mathbf{R})$ in real-space is obtained, one can readily construct the Hamiltonian at arbitrary $\mathbf{k}$ points in NAO basis sets,

$$
H_{\tilde{I} i, \tilde{J} j}(\mathbf{k})=\sum_{|\mathbf{R}|<=R_{\max }} e^{i \mathbf{k} \cdot \mathbf{R}} H_{\tilde{I}, \tilde{J} j}(\mathbf{R})
$$


The reason that the lattice summation in eq (21) can also be restricted below $R_{\max }$ is because the local part of the gKS Hamiltonian is more short-ranged than the HFX part. Now, given that the Hamiltonian matrix at arbitrary $\mathbf{k}$ points is readily available from eq (21), the band energies along desired paths in $\mathbf{k}$ space can be obtained by a one-shot diagonalization through non-SCF calculations. Compared to the plane-wave formalism, the diagonalization at the final step is rather inexpensive, due to the much reduced basis size in the NAO framework. Therefore, one does not need to invoke the band interpolation techniques here, ${ }^{102,103}$ as is usually within the plane-wave approach. Such a real-space algorithm is also of great advantage if very dense $\mathbf{k}$ grids are needed, e.g., when calculating the optical adsorption spectra.

To check the validity of our approach for HDF band structure calculations, in Fig. 11 we present the HSE06 band structures for $\mathrm{Si}$ and GaP crystals as obtained by ABACUS, in comparison with the corresponding FHI-aims ${ }^{51,55,57}$ results, which are taken as the reference here. The valence-only DZP basis sets $(2 s 2 p 1 d$ for $\mathrm{Si}$ and $\mathrm{P}$, and $2 s 2 p 2 d 1 f$ for Ga) are used in ABACUS calculations, whereas the so-called "tight" setting is used in FHI-aims calculations, corresponding to all-electron $4 s 3 p 2 d 1 f 1 g$ basis set for Si and $\mathrm{P}$, and $5 s 4 p 2 d 1 f$ basis set for Ga). Despite the different (pseudopotential versus all-electron) descriptions of core-valence interactions and different basis sizes, the valence and low-lying conduction bands (and hence the band gap) obtained using the two codes agree with each other rather well. The remaining discrepancy for the high-lying conduction bands is expected due to the relatively smaller basis size used in ABACUS calculations. The agreement will get further improved if one employs the TZDP basis sets in ABACUS calculations. A more comprehensive comparison study of the HSE06 band gaps obtained using different computer codes, as well as the influence of the basis sets, can be found in Ref. ${ }^{58}$ 

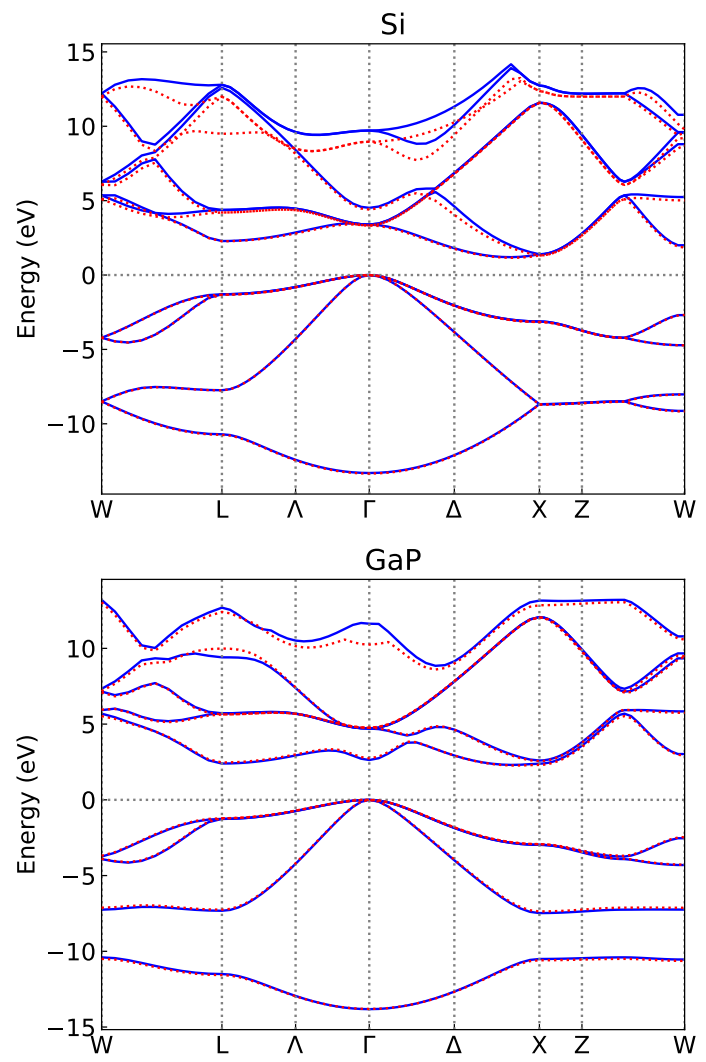

Figure 11: HSE06 electronic band structures of Si (upper panel, diamond structure) and GaP (lower panel, zinc blende structure) calculated using ABACUS (blue solid lines) and FHI-aims (red dashed lines) codes. The experimental lattice parameters are used for both materials. A $8 \times 8 \times 8 \mathrm{k}$ grid is used in the BZ integration, and the NAO basis sets used by the two codes in the present calculations can be found in the text. 


\section{Summary}

In summary, we presented an efficient, linear-scaling implementation for building the (screened) HFX matrix for periodic systems within the framework of NAO basis functions. The implementation was based on the LRI approximation, coupled with our own procedures for constructing the ABFs. The numerical accuracy of such an approximation for periodic HDF calculations has been systematically benchmarked in Ref. 58 .

In this work, we described the numerical details behind our implementation, in particular how we choose the loop structure over the atom pairs, and how we exploit the sparsity of the key matrices, including expansion coefficients, the (screened) Coulomb matrix, and the density matrix. In the latter case, a multi-level screening procedure is employed which ensures the insignificant elements of these matrices can be efficiently screened out as much as possible, leading to a linear-scaling build of the HFX matrix with a rather small prefactor. We discussed two parallel distribution schemes, which can be invoked to achieve the best load balance for computation time, or alternatively, to reduce the memory consumption when there is a shortage of memory. Benchmark calculations for Si crystal with supercells up to 4096 atoms confirms the linear-scaling behavior of the computation cost with respect to the system size, whereas calculations with increasing computing resources demonstrate the excellent parallel efficiency up to $10^{3} \mathrm{CPU}$ cores.

Our implementation was carried out in ABACUS, but the same techniques can be easily utilized by other local-orbital based computer code package. With our present implementation in ABACUS, HDF calculations for systems with a few thousand atoms can be routinely done with modest computing resources. We that expect our implementation will find important applications in disordered systems, defects, and heterostructures where large supercells are needed. 


\section{Acknowledgement}

We thank Liu Xiaohui, Chen Junshi, Shen Yu and Shi Rong for helpful discussions. The work is supported by the National Key Research and Development Program of China (Grant No. 2016YFB0201202) and National Natural Science Foundation of China (Grant Numbers 11774327, 11874335). The numerical calculations have been partly done in National Supercomputer Center in GuangZhou and partly on the USTC HPC facilities.

\section{A Appendix}

\section{A.1 Ordering of matrix multiplications to evaluate $\mathcal{H}^{\mathrm{X}}$ matrices}

The key step in building the HFX matrix is to evaluate the $\mathcal{H}^{\mathrm{x}}$ matrices, formally introduced in eq (9). There are four variants of them (cf. eq (7)), depending on where the ABFs are located, but all can be seen as the contributions to $\Sigma_{I i, J j}^{\mathrm{X}}$ from the the atom pair $\langle K, L\rangle$. They are formally given by $\mathcal{H}^{\mathrm{x}} \sim C V C D$, i.e., a sequence of matrix products involving $C, V, D$ matrices. Obviously, the actual order of performing the matrix multiplication is important here, since it will affect the overall computational cost.

The issue can be analyzed for a given set of four atoms - I, J, K, L. In this case, omitting the atomic indices, the $\mathcal{H}^{\mathrm{x}}$ matrices are rank-2 tensors and can be calculated as

$$
\mathcal{H}_{i j}^{\mathrm{X}}=\sum_{k l} \sum_{\alpha \beta} C_{i k}^{\alpha} V_{\alpha \beta} C_{j l}^{\beta} D_{k l}
$$

For the convenience of analysis, we assume that all atoms have the same number of AOs $\left(n_{\phi}\right)$ and ABFs $\left(n_{P}\right)$. Here we emphasize that $n_{\phi}$ and $n_{P}$ refer to the number of basis functions per atom (and not per unit cell). Therefore, in eq (22) $C$ is a $n_{P} \times n_{\phi} \times n_{\phi}$ 3rd-rank tensor, and $V$ and $D$ are, respectively, $n_{P} \times n_{P}$ and $n_{\phi} \times n_{\phi}$ matrices.

Given the expression in eq (22), one may recognize that there are five different ways of ordering the matrix multiplications. The computational costs associated with the five 
orderings are list in Table 1, among which the most efficient two are obviously $(C V)(C D)$ and $C((V C) D)$, due to the fact that $n_{P}$ is several times larger than $n_{\phi}$. After considering data structures adopted in the code, cache optimization, and the use of BLAS ${ }^{90-92}$ library, we finally chose $C((V C) D)$ as the matrix multiplication order in our implementation.

Table 1: computational cost of order of matrix multiplication

\begin{tabular}{cc}
\hline order of matrix multiplication & computational cost \\
\hline$(C V)(C D)$ & $n_{P}^{2} n_{\phi}^{2}+8 n_{P} n_{\phi}^{3}$ \\
$C((V C) D)$ & $n_{P}^{2} n_{\phi}^{2}+8 n_{P} n_{\phi}^{3}$ \\
$C(V(C D))$ & $4 n_{P}^{2} n_{\phi}^{2}+8 n_{P} n_{\phi}^{3}$ \\
$((C V) C) D$ & $n_{P} n_{\phi}^{4}+n_{P}^{2} n_{\phi}^{2}+4 n_{\phi}^{4}$ \\
$(C(V C)) D$ & $n_{P} n_{\phi}^{4}+n_{P}^{2} n_{\phi}^{2}+4 n_{\phi}^{4}$ \\
\hline
\end{tabular}

\section{References}

(1) Becke, A. D. Density-functional thermochemistry. III. The role of exact exchange. J. Chem. Phys 1993, 98, 5648.

(2) Perdew, J. P.; Schmidt, K. In Density Functional Theory and its Application to Materials ; Van Doren, V., Van Alsenoy, C., Geerlings, P., Eds.; AIP: Melville, NY, 2001.

(3) Hohenberg, P.; Kohn, W. Inhomogeneous Electron Gas. Phys. Rev. 1964, 136, B864.

(4) Kohn, W.; Sham, L. J. Self-Consistent Equations Including Exchange and Correlation Effects. Phys. Rev. 1965, 140, A1133.

(5) Seidl, A.; Görling, A.; Vogl, P.; Majewski, J. A.; Levy, M. Generalized Kohn-Sham schemes and the band-gap problem. Phys. Rev. B 1996, 53, 3764.

(6) Perdew, J. P.; Ernzerhof, M.; Burke, K. Rationale of mixing exact exchange with density functional approximations. J. Chem. Phys. 1996, 105, 9982. 
(7) Ernzerhof, M.; Scuseria, G. E. J. Chem. Phys. 1999, 110, 5029.

(8) Adamo, C.; Barone, V. J. Chem. Phys. 1999, 110, 6158.

(9) Zhao, Y.; Truhlar, D. G. The M06 suite of density functionals for main group thermochemistry, thermochemical kinetics, noncovalent interactions, excited states, and transition elements: two new functionals and systematic testing of four M06-class functionals and 12 other functionals. Theor. Chem. Acc. 2008, 120, 215-241.

(10) Chai, J.-D.; Head-Gordon, M. Systematic optimization of long-range corrected hybrid density functionals. J. Chem. Phys. 2008, 128, 084106.

(11) Heyd, J.; Scuseria, G. E.; Ernzerhof, M. Hybrid functionals based on a screened Coulomb potential. J. Chem. Phys. 2003, 118, 8207.

(12) Toulouse, J.; Colonna, F.; Savin, A. Long-rangeshort-range separation of the electronelectron interaction in density-functional theory. Phys. Rev. A 2004, 70, 062505.

(13) Iikura, H.; Tsuneda, T.; Yanai, T.; Hirao, K. A long-range correction scheme for generalized-gradient-approximation exchange functionals. J. Chem. Phys. 2001, 115, 3540 .

(14) Yanai, T.; Tew, D. P.; Handy, N. C. A new hybrid exchangecorrelation functional using the Coulomb-attenuating method (CAM-B3LYP). Chem. Phys. Lett. 2004, 393, 51.

(15) Vydrov, O. A.; Scuseria, G. E. Assessment of a long-range corrected hybrid functional. J. Chem. Phys. 2006, 125, 234109.

(16) Skone, J. H.; Govoni, M.; Galli, G. Separated Hybrid Functionals for Solids and Molecules. Phys. Rev. B.: Condens. Mater. Phys. 2016, 93, 235106.

(17) Shimazaki, T.; Asai, Y. Band Structure Calculations Based on Screened Fock Exchange Method. Chem. Phys. Lett. 2008, 466, 91-94. 
(18) Shimazaki, T.; Asai, Y. Energy band structure calculations based on screened HartreeFock exchange method: Si, AlP, AlAs, GaP, and GaAs. J. Chem. Phys. 2010, 132, 224105.

(19) Marques, M. A. L.; Vidal, J.; Oliveira, M. J. T.; Reining, L.; Botti, S. Density-Based Mixing Parameter for Hybrid Functionals. Phys. Rev. B.: Condens. Matter Mater. Phys. 2011, 83, 035119.

(20) Shimazaki, T.; Nakajima, T. Dielectric-dependent screened HartreeFock exchange potential and Slater-formula with Coulomb-hole interaction for energy band structure calculations. J. Chem. Phys. 2014, 141, 114109.

(21) Skone, J. H.; Govoni, M.; Galli, G. Self-consistent hybrid functional for condensed systems. Phys. Rev. B.: Condens. Mater. Phys. 2014, 89, 195112.

(22) He, J.; Franchini, C. Assessing the Performance of Self-Consistent Hybrid Functional for Band Gap Calculation in Oxide. J. Phys.: Condens. Matter 2017, 29, 454004.

(23) Kronik, L.; Stein, T.; Refaely-Abramson, S.; ; Baer, R. Excitation Gaps of FiniteSized Systems from Optimally Tuned Range-Separated Hybrid Functionals. J. Chem. Theory Comput. 2012, 8, 1515.

(24) Atalla, V.; Yoon, M.; Caruso, F.; Rinke, P.; Scheffler, M. Hybrid density functional theory meets quasiparticle calculations. Phys. Rev. B 2013, 88, 165112.

(25) Cui, Z.-H.; Wang, Y.-C.; Zhang, M.-Y.; Xu, X.; Jiang, H. Doubly Screened Hybrid Functional: An Accurate First-Principles Approach for Both Narrow- and Wide-Gap Semiconductors. J. Phys. Chem. Lett. 2018, 9, 2338-2345.

(26) Zhang, M.-Y.; Cui, Z.-H.; Wang, Y.-C.; Jiang, H. Hybrid functionals with systemdependent parameters: conceptual foundation and methodological developments. WIREs Comput. Mol. Sci. 2020, 
(27) Almlöf, J.; Faegri, Jr., K.; Korsell, K. Principles for a Direct SCF Approach to LCAOMO Ab-lnitio Calculations. J. Comput. Chem. 1982, 3, 385.

(28) Häser, M.; Ahlrichs, R. Improvements on the direct SCF method. J. Comput. Chem. 1989, 10, 104.

(29) Burant, J. C.; Scuseria, G. E.; Frisch, M. J. A linear scaling method for HartreeFock exchange calculations of large molecules. J. Chem. Phys. 1996, 105, 8969.

(30) Schwegler, E.; Challacombe, M. Linear scaling computation of the HartreeFock exchange matrix. J. Chem. Phys. 1996, 105, 2726.

(31) Schwegler, E.; Challacombe, M.; Head-Gordon, M. Linear scaling computation of the Fock matrix. II. Rigorous bounds on exchange integrals and incremental Fock build. J. Chem. Phys. 1997, 106, 9708.

(32) Ochsenfeld, C.; White, C. A.; Head-Gordon, M. Linear and sublinear scaling formation of Hartree-Fock-type exchange matrices. J. Chem. Phys. 1998, 109, 1663.

(33) Rudberg, E.; Rubensson, E. H.; ; Sałek, P. Kohn-Sham Density Functional Theory Electronic Structure Calculations with Linearly Scaling Computational Time and Memory Usage. J. Chem. Theory Comput. 2011, 7, 340.

(34) Pisani, C.; Dovesi, R. Exact-Exchange Hartree-Fock Calculations for Periodic Solids. I. Illustration of the Method. Int. J. Quantum Chem. 1980, XVII, 501.

(35) Pisani, C.; Dovesi, R.; Roetti, C. Hartree-Fock Ab Initio Treatment of Crystalline solids, Volume 48 of Lecture Notes in Chemistry Series; Springer Verlag: Berlin, 1988.

(36) Dovesi, R.; Orlando, R.; Erba, A.; Zicovich-Wilson, C. M.; Civalleri, B.; Casassa, S.; Maschio, L.; Ferrabone, M.; De La Pierre, M.; DArco, P.; Noël, Y.; Causà, M.; 
Rérat, M.; ; Kirtman, B. CRYSTAL14: A Program for the Ab Initio Investigation of Crystalline Solids. Int. J. Quan. Chem. 2014, 114, 1287.

(37) Guidon, M.; Schiffmann, F.; Hutter, J.; VandeVondele, J. Ab initio molecular dynamics using hybrid density functionals. The Journal of chemical physics 2008, 128, 214104.

(38) Guidon, M.; Hutter, J.; VandeVondele, J. Robust Periodic Hartree-Fock Exchange for Large-Scale Simulations Using Gaussian Basis Sets. J. Chem. Theory Comput. 2009, 5,3010 .

(39) Paier, J.; Diaconu, C. V.; Scuseria, G. E.; ad Joost VandeVondele, M. G.; Hutter, J. Accurate Hartree-Fock energy of extended systems using large Gaussian basis sets. Phys. Rev. B 2009, 80, 174114.

(40) Paier, J.; Marsman, M.; Hummer, K.; Kresse, G.; Gerber, I. C.; Ángyán, J. G. Screened hybrid density functionals applied to solids. J. Chem. Phys. 2006, 124, 154709 .

(41) Betzinger, M.; Friedrich, C.; Blügel, S. Hybrid functionals within the all-electron FLAPW method: Implementation and applications of PBE0. Phys. Rev. B 2010, $81,195117$.

(42) Wu, X.; Selloni, A.; Car, R. Order-N implementation of exact exchange in extended insulating systems. Physical Review B 2009, 79, 085102.

(43) Lin, L. Adaptively compressed exchange operator. Journal of chemical theory and computation 2016, 12, 2242-2249.

(44) Becke, A. Density-functional exchange-energy approximation with correct asymptotic behavior. Phys. Rev. A 1988, 38, 3098. 
(45) Lee, C.; Yang, W.; Parr, R. G. Development of the Colle-Salvetti correlation-energy formula into a functional of the electron density. Phys. Rev. B 1988, 37, 785-789.

(46) Perdew, J. P.; Burke, K.; Ernzerhof, M. Generalized Gradient Approximation Made Simple. Phys. Rev. Lett 1996, 77, 3865.

(47) Delly, B. From molecules to solids with the DMol ${ }^{3}$ approach. J. Chem. Phys. 2000, $113,7756$.

(48) Koepernik, K.; Eschrig, H. Full-potential nonorthogonal local-orbital minimum-basis band-structure scheme. Phys. Rev. B 1999, 59, 1743.

(49) Soler, J. M.; Artacho, E.; Gale, J. D.; García, A.; Junquera, J.; Ordejón, P.; SánchezPortal, D. The SIESTA method for ab initio order-N materials simulation. J. Phys.: Condens. Matter 2002, 14, 2745.

(50) Ozaki, T.; Kino, H.; Yu, J.; Han, M.; Kobayashi, N.; Ohfuti, M.; Ishii, F.; Ohwaki, T. User's manual of OpenMX, http://www.openmx-square.org, 2008.

(51) Blum, V.; Hanke, F.; Gehrke, R.; Havu, P.; Havu, V.; Ren, X.; Reuter, K.; Scheffler, M. Ab-initio molecular simulations with numeric atom-centered orbitals. Comp. Phys. Comm. 2009, 180, 2175.

(52) Li, P.; Liu, X.; Chen, M.; Lin, P.; Ren, X.; Lin, L.; Yang, C.; He, L. Large-scale ab initio simulations based on systematically improvable atomic basis. Comput. Mater. Sci. 2016, 112, 503.

(53) Shang, H.; Li, Z.; Yang, J. Implementation of Exact Exchange with Numerical Atomic Orbitals. J. Phys. Chem. A 2010, 114, 1039.

(54) Qin, X.; Shang, H.; Xiang, H.; Li, Z.; Yang, J. HONPAS: A Linear Scaling OpenSource Solution for Large System Simulations. Int. J. Quan. Chem. 2015, 115, 647. 
(55) Ren, X.; Rinke, P.; Blum, V.; Wieferink, J.; Tkatchenko, A.; Sanfilippo, A.; Reuter, K.; Scheffler, M. Resolution-of-identity approach to HartreeFock, hybrid density functionals, RPA, MP2 and GW with numeric atom-centered orbital basis functions. New J. Phys. 2012, 14, 053020.

(56) Ihrig, A. C.; Wieferink, J.; Zhang, I. Y.; Ropo, M.; Ren, X.; Rinke, P.; Scheffler, M.; Blum, V. Accurate localized resolution of identity approach for linear-scaling hybrid density functionals and for many-body perturbation theory. New J. Phys. 2015, 17, 093020.

(57) Levchenko, S. V.; Ren, X.; Wieferink, J.; Johanni, R.; Rinke, P.; Blum, V.; Scheffler, M. Hybrid functionals for large periodic systems in an all-electron, numeric atomcentered basis framework. Comp. Phys. Comm. 2015, 192, 60.

(58) Lin, P.; Ren, X.; He, L. Accuracy of Localized Resolution of the Identity in Periodic Hybrid Functional Calculations with Numerical Atomic Orbitals. J. Phys. Chem. Lett. 2020, 11, 3082 .

(59) Lu, J.; Yin, L. Compression of the electron repulsion integraltensor in tensor hypercontraction format with cubic scaling cost. J. Comput. Phys. 2015, 302, 329.

(60) Qin, X.; Hu, W.; Yang, J. Interpolative separable density fitting decomposition for accelerating Hartree-Fock exchange calculations within numerical atomic orbitals. J. Phys. Chem. A 2020, 124, 5664.

(61) Feyereisen, M.; Fitzgerald, G.; Komornicki, A. Use of approximate integrals in ab initio theory, An application in MP2 energy calculations. Chem. Phys. Lett. 1993, 208,359 .

(62) Vahtras, O.; Almlöf, J.; Feyereisen, M. W. Integral approximations for LCAO-SCF calculations. Chem. Phys. Lett. 1993, 213, 514. 
(63) Weigend, F.; Häser, M.; Patzelt, H.; Ahlrichs, R. RI-MP2: optimized auxiliary basis sets and demonstration of efficiency. Chem. Phys. Lett. 1998, 294, 143.

(64) Whitten, J. L. Coulomb potential energy integrals and approximations. J. Chem. Phys. 1973, 58, 4496.

(65) Dunlap, B. I.; Connolly, J. W. D.; Sabin, J. R. On some approximations of X $\alpha$ method. J. Chem. Phys 1979, 71, 3396.

(66) Dunlap, B. I.; Rösch, N.; Trickey, S. Variational fitting methods for electronic structure calculations. Mol. Phys 2010, 108, 3167.

(67) Weigend, F. A fully direct RI-HF algorithm: Implementation, optimised auxiliary basis sets, demonstration of accuracy and efficiency. Phys. Chem. Chem. Phys. 2002, 4, 4285 .

(68) Eshuis, H.; Yarkony, J.; Furche, F. Fast computation of molecular random phase approximation correlation energies using resolution of the identity and imaginary frequency integration. J. Chem. Phys. 2010, 132, 234114.

(69) Del Ben, M.; Hutter, J.; VandeVondele, J. Electron Correlation in the Condensed Phase from a Resolution of Identity Approach Based on the Gaussian and Plane Waves Scheme. J. Chem. Theo. Comput. 2013, 9, 2654.

(70) Merlot, P.; Kjrgaard, T.; Helgaker, T.; Lindh, R.; Aquilante, F.; Reine, S.; Pedersen, T. B. Attractive ElectronElectron Interactions within Robust Local Fitting Approximations. J. Comput. Chem. 2013, 34, 1486.

(71) Billingsley II, F. P.; Bloor, J. E. Limited Expansion of Diatomic Overlap (LEDO): A Near?Accurate Approximate Ab Initio LCAO MO Method. I. Theory and Preliminary Investigations. J. Chem. Phys. 1971, 55, 5178. 
(72) Pisani, C.; M, M. B.; Capecchi, G.; Casassa, S.; Dovesi, R.; Maschio, L.; ZicovichWilson, C.; Schütz, M. Local-MP2 electron correlation method for nonconducting crystals. J. Chem. Phys. 2005, 122, 094113.

(73) Pisani, C.; Maschio, L.; Casassa, S.; Halo, M.; Schütz, M.; Usvyat, D. Periodic Local MP2 Method for the Study of Electronic Correlation in Crystals: Theory and Preliminary Applications. J. Comput. Chem. 2008, 29, 2113.

(74) Sodt, A.; Subotnik, J. E.; Head-Gordon, M. Linear scaling density fitting. J. Chem. Phys. 2006, 125, 194109.

(75) Sodt, A.; Head-Gordon, M. Hartree-Fock exchange computed using the atomic resolution of the identity approximation. J. Chem. Phys. 2008, 128, 104106.

(76) Reine, S.; Tellgren, E.; Krapp, A.; Kj?rgaard, T.; Helgaker, T.; Jansik, B.; H?st, S.; Salek, P. Variational and robust density fitting of four-center two-electron integrals in local metrics. J. Chem. Phys. 2008, 129, 104101.

(77) Wirz, L. N.; Reine, S. S.; Pedersen, T. B. On Resolution-of-the-Identity Electron Repulsion Integral Approximations and Variational Stability. J. Comput. Chem. 2017, $13,4897$.

(78) The ABACUS software webpage: http://abacus.ustc.edu.cn.

(79) Chen, M.; Guo, G.-C.; He, L. Systematically improvable optimized atomic basis sets for ab initio calculations. J. Phys.: Condens. Matter 2010, 22, 445501.

(80) Hamann, D.; Schlüter, M.; Chiang, C. Norm-conserving pseudopotentials. Physical Review Letters 1979, 43, 1494.

(81) Talman, J. D. Numerical Fourier and Bessel transforms in logarithmic variables. $J$. Comp. Phys. 1978, 29, 35-48. 
(82) Talman, J. D. Numerical calculation of four-center Coulomb integrals. J. Chem. Phys. 1984, 80, 2000-2008.

(83) Talman, J. D. Numerical methods for multicenter integrals for numerically defined basis functions applied in molecular calculations. Int. J. Quant. Chem. 2003, 93, $72-90$.

(84) MPI. https://www.mpi-forum.org/, 2020.

(85) Gygi, F.; Baldereschi, A. Self-consistent Hartree-Fock and screened-exchange calculations in solids: Application to silicon. Phys. Rev. B 1986, 34, 4405.

(86) Spencer, J.; Alavi, A. Efficient calculation of the exact exchange energy in periodic systems using a truncated Coulomb potential. Phys. Rev. B 2008, 77, 193110.

(87) Des Cloizeaux, J. Energy Bands and Projection Operators in a Crystal: Analytic and Asymptotic Properties. Phys. Rev. 1959, 115, 809.

(88) Des Cloizeaux, J. Energy Bands and Projection Operators in a Crystal: Analytic and Asymptotic Properties. Phys. Rev. 1964, 135, A685.

(89) He, L.; Vanderbilt, D. Exponential decay properties of Wannier functions and related quantities. Physical Review Letters 2001, 86, 5341.

(90) Lawson, C. L.; Hanson, R. J.; Kincaid, D. R.; Krogh, F. T. Basic linear algebra subprograms for Fortran usage. 1977,

(91) Dongarra, J. J.; Du Croz, J.; Hammarling, S.; Hanson, R. J. An extended set of FORTRAN basic linear algebra subprograms. ACM Transactions on Mathematical Software (TOMS) 1988, 14, 1-17.

(92) Dongarra, J. J.; Cruz, J. D.; Hammarling, S.; Duff, I. S. Algorithm 679: A set of level 3 basic linear algebra subprograms: model implementation and test programs. $A C M$ Transactions on Mathematical Software (TOMS) 1990, 16, 18-28. 
(93) Häser, M.; Ahlrichs, R. Improvements on the direct SCF method. Journal of Computational Chemistry 1989, 10, 104-111.

(94) OpenMP. https://www .openmp.org/, 2020.

(95) Kan, R.; George, A. Machine scheduling problems : classification, complexity and computations. 1976,

(96) Lenstra, J. K.; Kan, A. R.; Brucker, P. Annals of discrete mathematics; Elsevier, 1977; Vol. 1; pp 343-362.

(97) Graham, R. L. Bounds for certain multiprocessing anomalies. Bell System Technical Journal 1966, 45, 1563-1581.

(98) Graham, R. L. Bounds on multiprocessing timing anomalies. SIAM journal on Applied Mathematics 1969, 17, 416-429.

(99) MacQueen, J., et al. Some methods for classification and analysis of multivariate observations. Proceedings of the fifth Berkeley symposium on mathematical statistics and probability. 1967; pp 281-297.

(100) Krukau, A. V.; Vydrov, O. A.; Izmaylov, A. F.; Scuseria, G. E. Influence of the exchange screening parameter on the performance of screened hybrid functionals. The Journal of chemical physics 2006, 125, 224106.

(101) Liu, Y.; Ren, X.; He, L. A DFT study of energetic and structural properties of a full turn of A-form DNA under relaxed and stretching conditions. The Journal of Chemical Physics 2019, 151, 215102.

(102) Pickett, W. E.; Krakauer, H.; B.Allen, P. Smooth Fourier interpolation of periodic functions. Phys. Rev. B 1988, 38, 2721.

(103) Shirley, E. L. Optimal basis sets for detailed Brillouin-zone integrations. Phys. Rev. B 1996, 54, 16464. 


\section{Graphical TOC Entry}

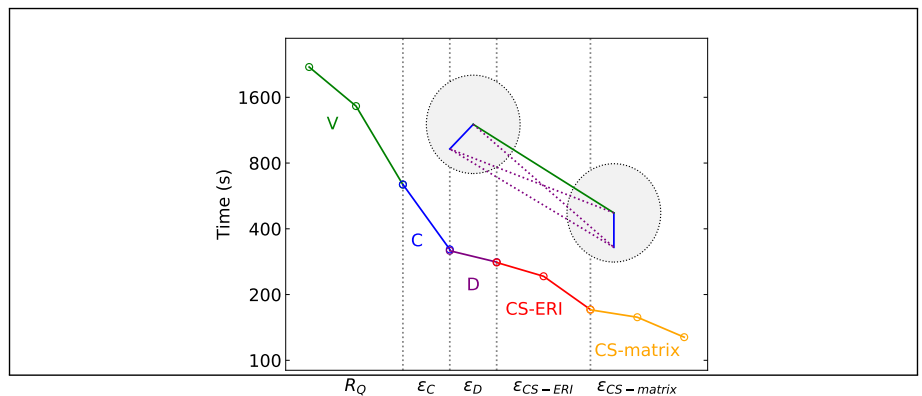

